\title{
Linking the Pilot Structural Model and Pilot Workload
}

\author{
Edward Bachelder ${ }^{1}$, Ronald Hess ${ }^{2}$, Bimal Aponso ${ }^{3}$, and Martine Godfroy-Cooper ${ }^{4}$ \\ San Jose State University/US Army Aviation Development Directorate/NASA Ames Research Center, Moffett Field, \\ CA, 94035, USA, University of California Davis, Davis, CA 95616
}

\begin{abstract}
Behavioral models are developed that closely reproduced pulsive control response of two pilots using markedly different control techniques while conducting a tracking task. An intriguing find was that the pilots appeared to: 1) produce a continuous, internallygenerated stick signal that they integrated in time; 2) integrate the actual stick position; and 3) compare the two integrations to either issue or cease a pulse command. This suggests that the pilots utilized kinesthetic feedback in order to sense and integrate stick position, supporting the hypothesis that pilots can access and employ the proprioceptive inner feedback loop proposed by Hess' pilot Structural Model (Ref. 1). A Pilot Cost Index was developed, whose elements include estimated workload, performance, and the degree to which the pilot employs kinesthetic feedback. Preliminary results suggest that a pilot's operating point (parameter values) may be based on control style and index minimization.
\end{abstract}

\section{Nomenclature}

$\begin{array}{ll}Y_{p} & =\text { pilot describing function } \\ Y_{c} & =\text { controlled element transfer function } \\ \tau & =\text { pilot time delay } \\ K & =\text { pilot gain } \\ z & =\text { pilot lead frequency } \\ \delta & =\text { pilot control input } \\ e & =\text { displayed error } \\ C M & =\text { Crossover Model }\end{array}$

\section{Introduction}

$\mathrm{T}$

he earliest study of the human operator as a linear servomechanism is that of Tustin (Ref. 2) who proposed that, despite amplitude nonlinearities, temporal discontinuities and haphazard fluctuations, there might be an "appropriate linear law" that would describe the main part of the operator's behaviour. Insight from servomechanical design led McRuer (Ref. 3) to develop the ubiquitous human crossover model (CM), which within its framework accounts for how, and why, the human operator adapts to the controlled plant dynamics during compensatory tracking. With the $C M$, a variable pilot time delay can be used to explain phenomena such as increased high-frequency phase lag associated with increased amounts of error lead equalization. Similarly, ratchet (sustained high frequency, small amplitude pilot-vehicle system oscillations) can be ascribed to variable neuromuscular damping, as investigated by Bachelder (Ref. 4). Smith (Ref. 5) first proposed the role of inner-loop rate feedback to support control of the error loop during compensatory tracking, whereby the rate of the controlled system's output was visually sensed by the operator. This manner of feedback was subject to two key constraints. The first being the bandwidth and noise characteristics associated with human visual sensing of rate, and the second is low ratio of disturbance-to-system output, without which the error rate sensed by the operator will not correspond to system output rate. A realizable method for sensing rate for use with inner-loop feedback was posed by Hess (Ref. 6), whereby the operator employs kinesthetic sensing of control rate and an internal model of system response to that rate. This approach was later incorporated into Hess' Structural Model of the human pilot (Refs. 1, 7). In 1969 Gaines (Ref. 8) wrote "Models capable of representing behaviour over large domains are particularly important in

\footnotetext{
${ }^{1}$ Senior Research Engineer, Member AIAA, edward.n.bachelder@nasa.gov.

${ }^{2}$ Professor, Dept. of Mechanical and Aeronautical Engineering, Fellow AIAA, rahess@, ucdavis.edu

${ }^{3}$ Branch Chief, Aviation Systems Division, Associate Fellow AIAA, bimal.1.aponso@nasa.gov

${ }^{4}$ Senior Research Scientist, NASA Ames Research Center, martine.godfroy-1@,nasa.gov
}

1

American Institute of Aeronautics and Astronautics 
the study of learning systems where the mode of behaviour is expected to vary widely with experience. A variety of models is required, and within the modelling schemata there must be scope for a sufficient variety to provide adequate matches during all phases of learning." Building on Krendel and McRuer's (Ref. 9) successive organization of perception (SOP) model for tracking skill development, Hess' pilot Structural Model provides a concise, integrated architecture for describing compensatory, pursuit, precognitive, and off-nominal behavior.

While human operator modeling has provided key methods for interpreting and evaluating pilot response, prediction of a pilot's operating point (i.e. the values of a model's parameters) using the classical control theory described above has not enjoyed much success, producing instead a set of general guidelines and rules. The development of modern control theory and linear quadratic Gaussian techniques in the early 1970s led to attempts to model the human pilot with an optimal regulator, filter, and estimator. This approach was mainly employed to predict pilor ratings by minimizing an objective or cost function that was judged to reflect the key variables affecting handling qualities ratings. The STI Optimal Control Model (Ref. 10) defined the cost function using error and control rate, where error captured the effects of performance and control rate captured the effects of workload on the rating. However, the pilot model frequency response did not resemble shapes observed from historical data and the the classical models. Also examined in Ref. 10 was a sub-optimal control model which specified the form of the human pilot using the gain, lead, and time delay of McRuer's crossover model. However, the performance index used for this analysis comprised of error and control amplitude (rather than control rate), and the predicted pilot models invariably employed pure lead (unrealistic human behavior) instead of first-order lead. Hess (Ref. 11) demonstrated good correlation between predicted and actual ratings, however this approach was subject to the following limitations:

1. A precise mathematical description of aircraft and display dynamics driving the tracking task was required to scale the control rate and error.

2. The tasks investigated were restricted to attitude tracking, and required that the vehicle dynamics could be represented in the form typical of aircraft pitch response where higher-order aircraft dynamics were linearized to fit this form.

3. The metric was extremely sensitive to the derived equivalent time delay $\tau_{\mathrm{D}}$.

The optimal pilot model suffers from three deficiencies. First, optimal pilot models are complex, both in the order of the predicted pilot describing function and in their implementation. Due to the nature of linear quadratic Gaussian theory, the predicted pilot describing function has an order of at least twice that of the aircraft dynamics. In Ref. 12 a sixth-order pilot was computed to control acceleration command dynamics $\left(1 / \mathrm{s}^{2}\right)$. This is not consistent with the gain, lead, lag, and delay observed in operator behavior, nor lends to an intuitive grasp of the pilot's control policies. An example of unrealistic modeling is when an aircraft's second order short period dynamics are effectively 'notched out' by the optimization, which is not achievable in human control. The second deficiency is over-parameterization. When implementing an optimal pilot model, the engineer has to make numerous parameter choices, such as noise intensities, performance index weightings, and forcing function form and intensity - the resulting pilot describing function is very sensitive to these choices. The third drawback with the optimal pilot model is that nonlinearities introduced by the pilot (such as pulsing technique) cannot be represented and accounted for.

The work presented here extends Hess' Structural Model (which is rooted in classical control) to account for and reproduce pilot compensatory behavior when different control styles are used. In doing so, it seeks to provide insight into the pilot's internal prioritization (or costing) that governs the parameter settings. Finally, examples are given of how pilot control technique (i.e. pulsive versus continuous) appears to influence the interplay between internal costing and parameter setting, ultimately affecting the operating point of the parameter set.

\section{Structural Model of the Human Pilot}

In Ref. 6 Hess proposed a model (see Figure 1) for human compensatory tracking whose essential features included an outer loop operating on error (e), an inner feedback loop operating on stick position $(\delta)$, pilot elements that equalize the error and stick signals (respectively $Y p_{e}, Y p_{\dot{m}}$ ), a pilot element $Y p_{n}$ generating the neuromuscular force of the particular limb which drives the manipulator, the manipulator dynamics $Y_{\delta}$ that produces the vehicle input from the force command, a controlled element $Y_{c}$ representing the vehicle dynamics, and the display element

$Y_{d}$ that transforms the physical system error to the displayed error being tracked. A disturbance $d$ is added to the vehicle output $m$, and the negative of this is the error that the operator is attempting to null.

2

American Institute of Aeronautics and Astronautics 
From Ref. 3 the neuromuscular element can be approximated by Eqn (1)

$$
Y_{P n}=\frac{\omega_{n}^{2}}{\left(S^{2}+2 \zeta_{n} \omega_{n}+\omega_{n}^{2}\right)}
$$

With the inner loop of Figure 1 closed, the simple quadratic form for $Y p_{n}$ can exhibit the key features of measured high-frequency human controller dynamics, namely, a typically subcritical damping ratio $\zeta n_{C L}$, and a minimum second-order amplitude fall-off beyond the undamped natural frequency $\omega n_{C L}$. The injected remnant signal $n_{e}$ is included to account for nonlinearities and/or time variations in quasilinear fashion.

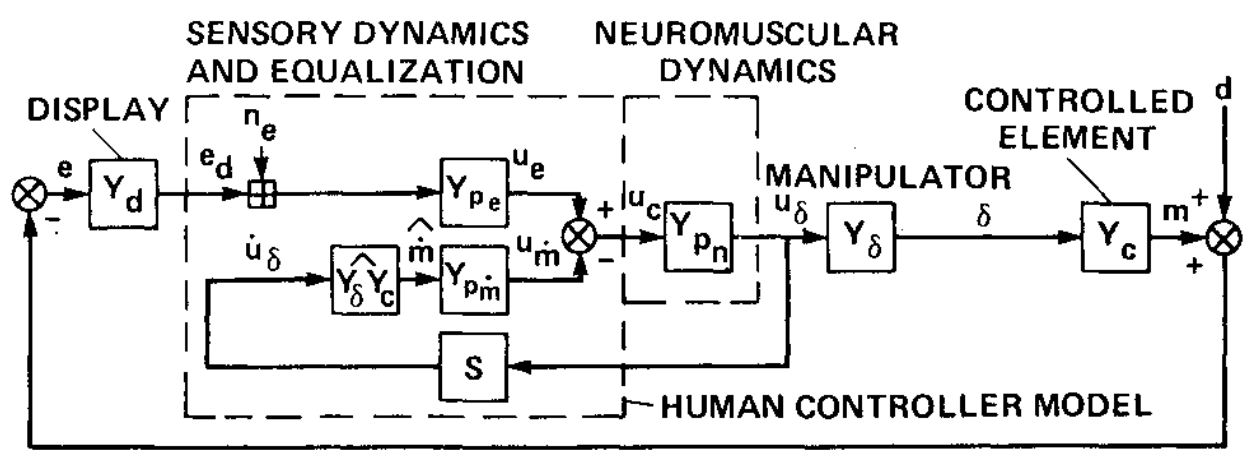

Figure 1. Quasilinear dual-loop model of the human controller (Ref. 6).

The main function of the inner-loop is to provide vehicle output rate feedback and improve stability and precision. Hess proposed the neuromuscular force output is sensed and transformed into an estimate of vehicle output rate using an internal model of the manipulator and vehicle dynamics suite. For acceleration command dynamics, this process effectively requires the pilot to integrate the force output (in the dual-loop model shown the pilot first senses the force rate, and integrates this twice). Hess hypothesized that proportional or derivative control in the feedback loop can be conducted using direct sensing from the muscle spindles and Golgi tendon organs (Ref. 13), but integral control does not have analogous sensing and requires the operator to employ higher level cognition. Thus when operating acceleration command systems, the pilot will tend to generate a pulsive force output rather than a continuous one since cognitively integrating the former is less difficult. "Ease of integrability" can be generally interpreted in a physiological sense as applying to those waveforms whose integration requires a minimum of higher-level activity in the central nervous system (Ref. 14).

As an operator becomes familiar with the vehicle and manipulator, the transformation between force output and vehicle output rate should reduce to a one-step process. In his revised Structural Model (Ref. 15), Hess reflects this simplification with a proprioceptive feedback element, $Y_{P F}$, shown in Figure 2. This element receives stick position, and depending on the vehicle dynamics in the vicinity of the crossover frequency $Y_{P F}$ will assume one of the forms shown in Table 1.

Table 1. Proprioceptive feedback element $Y_{P F}$ form.

\begin{tabular}{|c|c|c|c|}
\hline Vehicle Dynamics & $\mathrm{K}_{\mathrm{v}}$ & $\mathrm{K}_{\mathrm{v}} / \mathrm{s}$ & $\mathrm{K}_{\mathrm{v}} / \mathrm{s}^{2}$ \\
\hline $\mathbf{Y}_{\mathbf{P F}}$ & $\mathrm{K}_{\delta}(\mathrm{s}+\mathrm{a})$ & $\mathrm{K}_{\delta}$ & $\mathrm{K}_{\delta} /(\mathrm{s}+\mathrm{a})$ \\
\hline
\end{tabular}




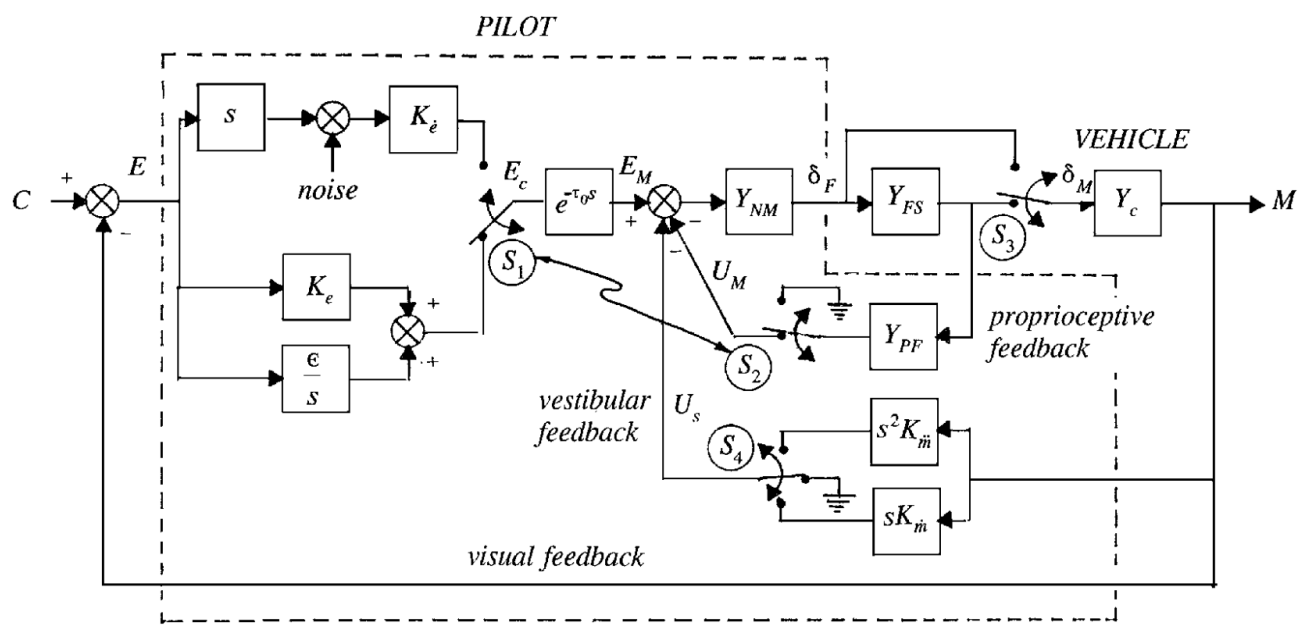

Figure 2. Structural Model of the Human Pilot (Ref. 15).

A gain $K_{e}$ serves as the pilot element acting on error, and the neuromuscular and feel system elements are respectively denoted by $Y_{N M}$ and $Y_{F S}$. While the various other details of the Structural Model (Figure 2) are described in Ref. 15, an element of key importance is the central processing time delay $\tau_{0}$, which for a given pilot can be approximated as vehicle-invariant. This represents a major simplification compared to the $C M$ 's effective time delay, which was used to explain (but could not predict) task and vehicle-dependent high frequency phase loss.

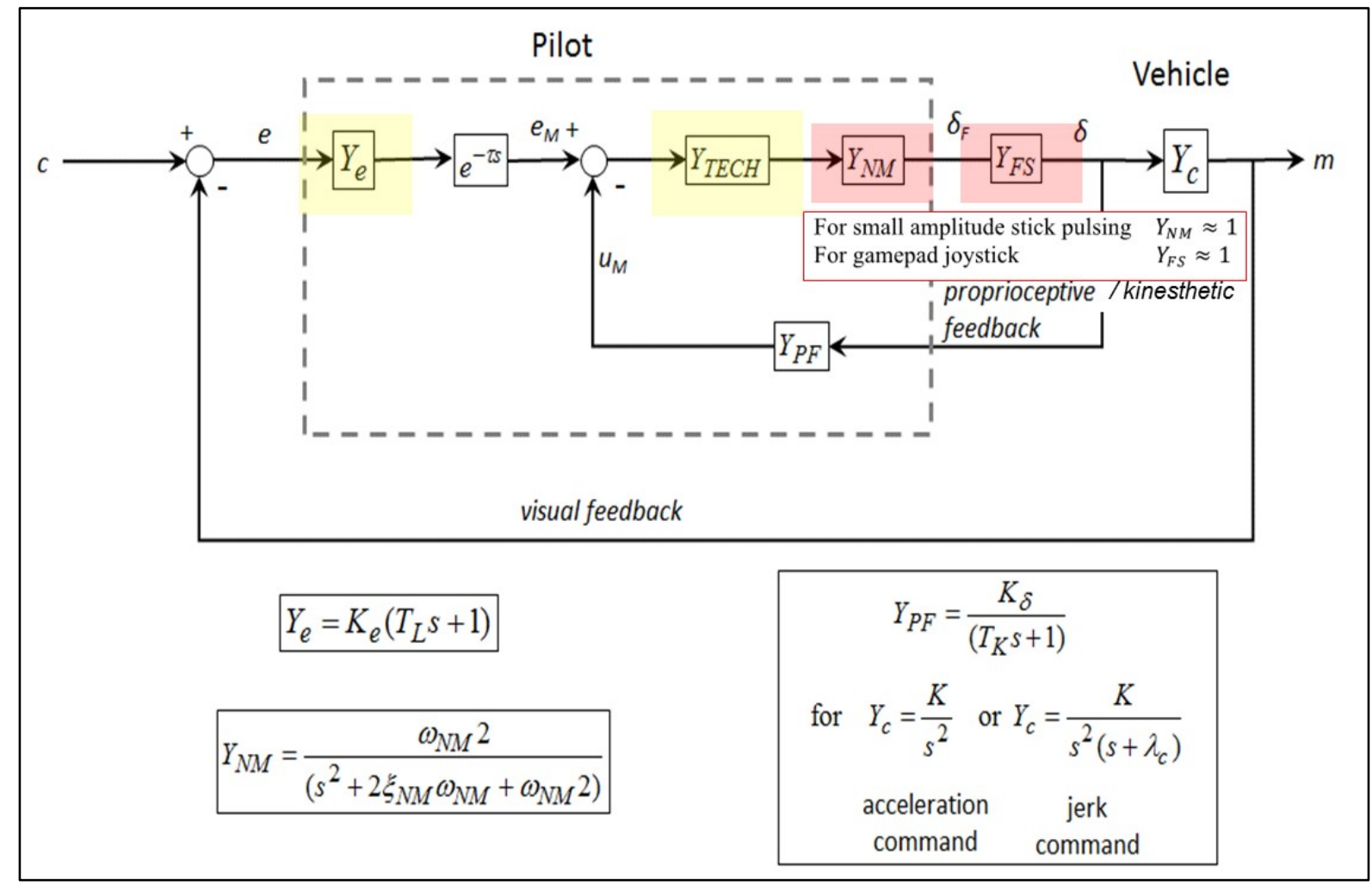

Figure 3. Pilot Structural Model simplified for fixed-base tracking.

Figure 3 reduces the Structural Model to the components relevant for fixed-base compensatory control (hence the vestibular paths are removed), with two modifications. The pilot element operating on the error channel is now 
represented by $Y_{e}$, and a pilot element $Y_{T E C H}$ has been added just prior to $Y_{N M}$. As shown in Figure 3, $Y_{e}$ is a firstorder lead instead of a pure gain, which gives error equalization more adaptation and appeared in this form in earlier versions of the Structural Model. $Y_{T E C H}$ represents the logic generating a pilot's non-linear control technique, which can be a set of conditionals and the supporting computations. The vehicle dynamics used in the following example cases will either be acceleration or jerk command dynamics, and $Y_{P F}$ for both will be a first-order lag. Later it will be shown that when the pilot employs pulsing, $Y_{N M}$ effectively becomes unity. Furthermore, for the controller used (a mini-joystick gamepad), it will be demonstrated that the feel system $Y_{F S}$ can also be approximated as unity when pulsing is utilized.

\section{Workload Experiment}

\section{A. Cognitive Workload}

Cognitive workload is the general term used to describe the mental cost of accomplishing task requirements (Ref. 16). Workload is a function of task demands placed on the human operator and the capacity of the operator to meet those demands (Ref. 17). High levels of workload occur when task demands exceed operator capacity. Many subjective procedures exist to measure mental workload, including the Cooper-Harper Scale (Ref. 18), the Bedford Scale (Ref. 19) which is a modification of the Cooper-Harper Scale, the SWAT (Subjective Assessment Technique) (Ref. 20) and the NASA-TLX (Task Load Index) (Ref. 21).

The Bedford scale is a unidimensional scale of 1 to 10 that uses a decision tree to examine whether the task can successufully be completed, and whether the level of workload is tolerable and satisfactory without reduction. The Bedford scale was selected for this project for its ease of administration and its effectiveness of assessing an operator's spare capacity.

This study used data from a piloted simulation experiment that was designed to examine factors affecting pilot's cognitive workload associated with vehicle control. The next section describes the experiment and summarizes the results.

\section{B. Experimental Protocol}

Four command vehicle dynamics (proportional, rate, acceleration, jerk), vehicle gains (vehicle sensitivity to input), and display gains (display sensitivity to error) were used with a lateral station-keeping task using a compensatory display, where a random forcing function continuously disturbed ownship's position.

Figure $4 \mathrm{a}$ shows a schematic of the station-keeping task and the display, pilot and vehicle components of the closed-loop system. Figure $4 \mathrm{~b}$ gives the range of conditions within each component that were tested. The jerk condition for the vehicle dynamics (fourth condition listed for $Y_{V}$ ), contains a pole $p$ whose location was varied. Twenty-three display configurations were tested with each subject using various combinations of the conditions. Since pilot proficiency was not a factor in this experiment, pilots were given two practice of each vehicle dynamic type (proportional, rate, acceleration, jerk) prior to testing.

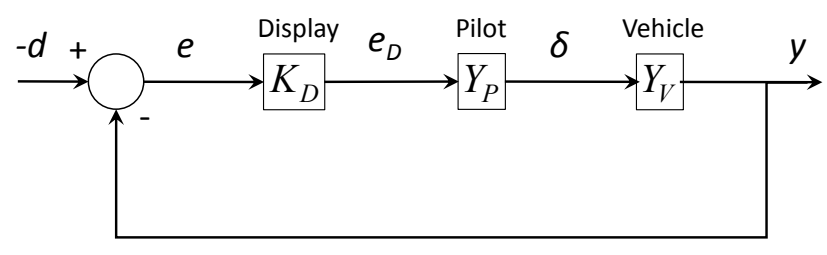

a.

$$
\begin{gathered}
Y_{V}=\left\{K_{V}, \frac{K_{V}}{s}, \frac{K_{V}}{s^{2}}, \frac{K_{V}}{(s+p) s^{2}}\right\} \\
K_{V}=\{5,10,30,60\} \quad p=\{1,2,5\} \\
K_{D}=\{0.5,2,4,8,32\}
\end{gathered}
$$

Figure 4. Station-keeping task. a) Pilot, display, and vehicle elements; b) Range of conditions for display and vehicle elements.

One may be tempted to think that the display gain and the vehicle gain are effectively interchangeable and the same from the pilot's vantage point. The following example will serve to disprove this common misnomer. If the pilot's input is zero, the disturbance is perceived through the display gain - the vehicle gain does not come into play at all. Based on his/her control activity and quiescence, a pilot learns to decouple the effects of the display gain from the vehicle gain - thus decoupling aircraft motion due to disturbance from pilot-commanded vehicle motion. 
Four right-handed male participants took part in the study. Three were Experimental Test Pilots (graduates of Navy Test Pilot School) with 1,900, 1,900, and 2,450 rotary wing flight hours. The fourth participant had logged 800 hours of rotary wing flight time. Ownship error relative to the hover location was displayed on a laptop monitor (see Figure 5), and the pilot attempted to minimize the error using a gaming joystick. The Bedford rating scale was used to subjectively score each pilots spare capacity at the end of each 60-second tracking run. Dependent variables were: stick position, rate and acceleration, stick position reversals, display error, rate, and acceleration. The positional disturbances imposed on the helicopter were designed to be both realistic and a diagnostic probe for pilot control behavior. Composed of a sum of 11 non-harmonically-related sine waves, the disturbance was perceived by the pilot as a random process - the result, however, was that the pilot's control response power resided largely at the same frequencies contained in the input disturbances. The disturbance time history is shown in Figure 5.

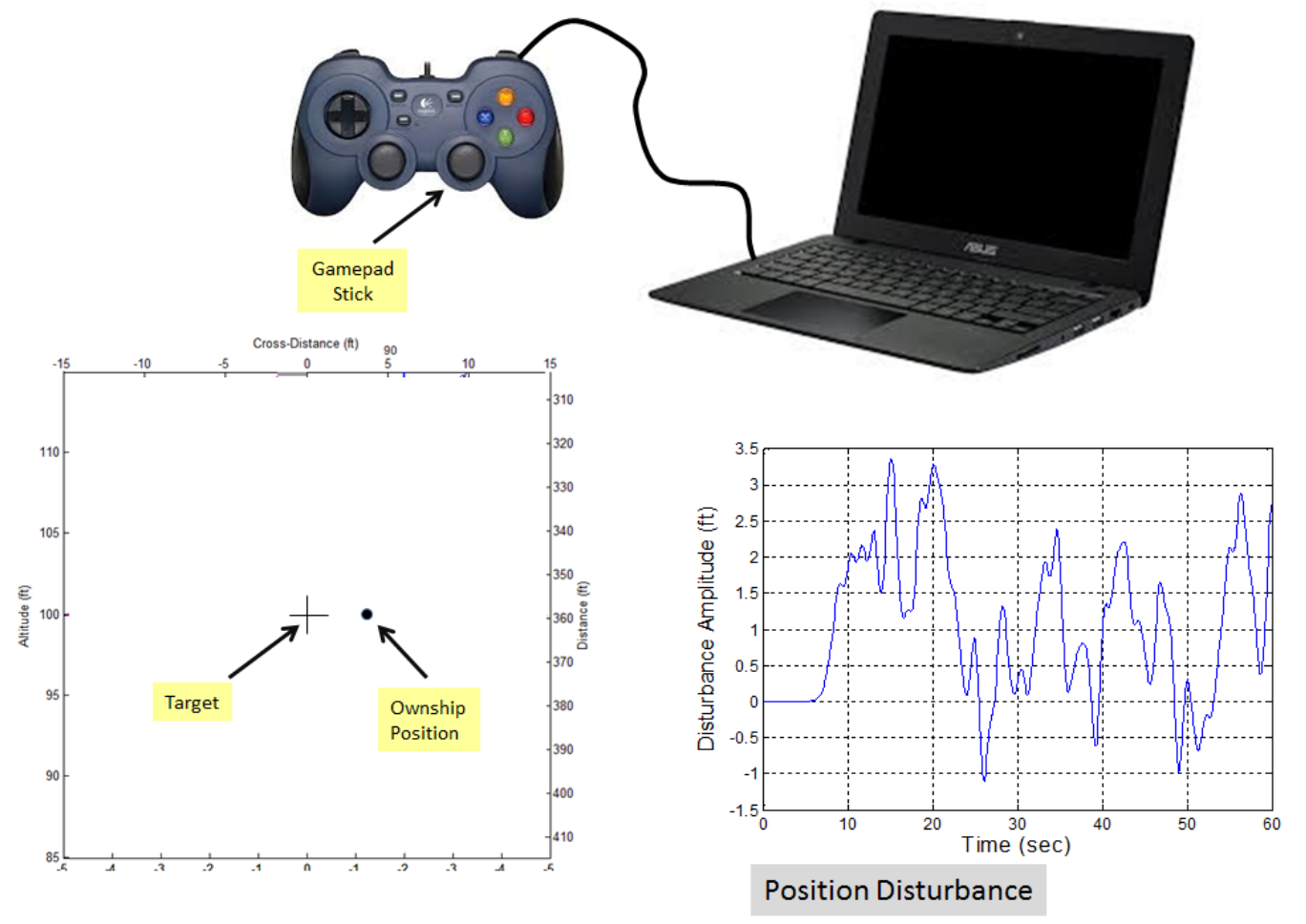

Figure 5. Simulation environment.

\section{Gamepad Use and Characteristics}

The right joystick of a Logitech F310 gamepad was used for the tracking task. The gamepad was held in the hands resting on the user's lap. Joystick control was conducted by thumb motion (in order to move the stick left/right the thumb must both extend-rotate left/retract-rotate right). Thus left motion may have been more easily effected than right motion, since moving right meant working against the limb's resistance. Maximum displacement of the joystick from center position to full left or righ was $0.8 \mathrm{~cm}$ (about $1 / 4$ inch).

A high resolution digital scale was used to measure the static force as a function of displacment, and this mapping is given in Figure 6a. A breakout force $\left(\mathrm{F}_{\mathrm{B}}\right)$ of 50 grams was required to move the joystick from neutral position, and the resistive force increased appproximately linearly to 110 grams at full deflection. On returning to the neutral position the force was approximately 50 grams throughout. A similar force-displacement profile is observed with the EH-60L cyclic, shown in Figure 6b (data obtained from US Army Aviation Development Directorate). The difference in slope between the advancing and return paths is attributed to friction. 


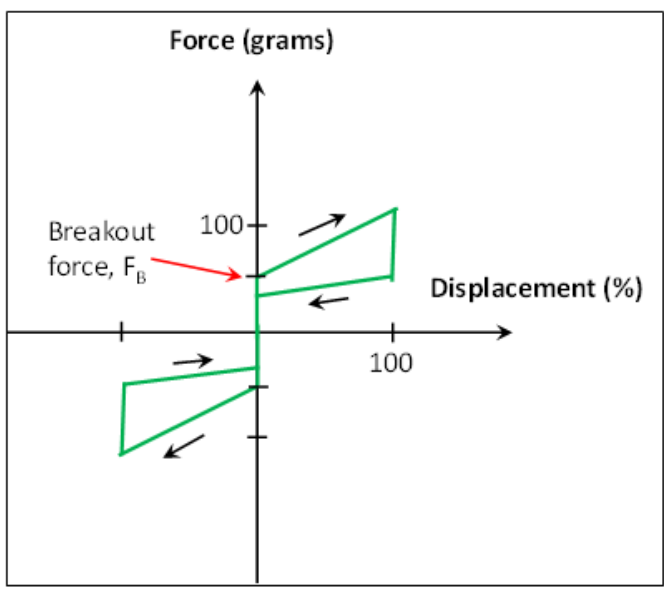

a.

Figure 6. Displacement vs. force: a) Gamepad joystick; b) EH-60L cyclic.

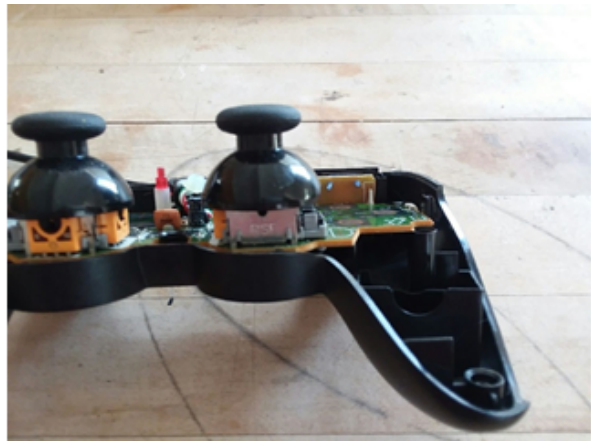

a.

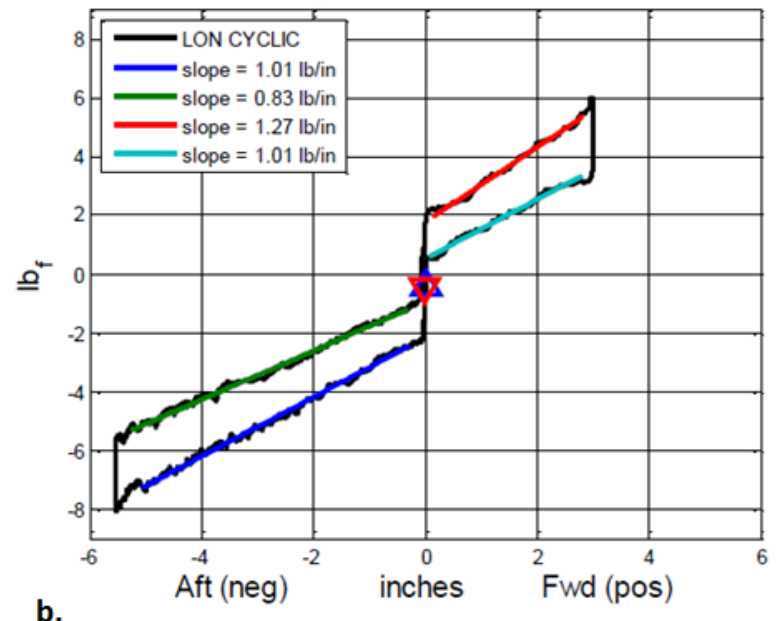

b.

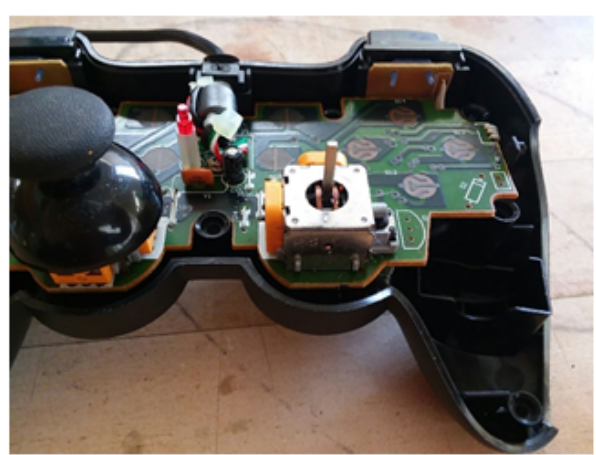

b.

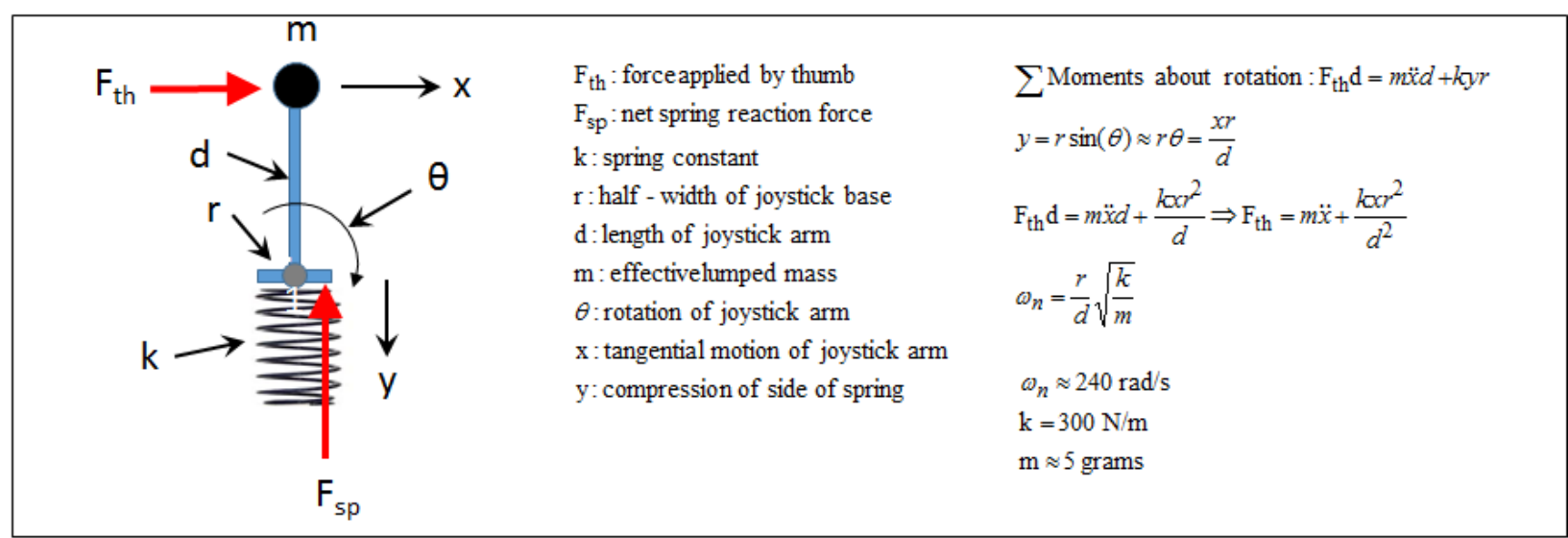

C.

\section{Figure 7. a) Gamepad joysticks without top cover; b) Right joystick cap removed; c) Moment diagram of joystick.}

In Figure 7a the gamepad top cover has been removed to expose both joysticks, and in Figure $7 \mathrm{~b}$ the right joystick cap has also been removed to show the lever arm attached to the two-axis controller. Inside the controller is a spring on which the circular base of the vertical arm sits. A simplified diagram of the mechanism and the moments summed about the arm's point of rotation is given in Figure 7c. The damped frequency of the joystick after release from full deflection is approximately $240 \mathrm{rad} / \mathrm{sec}$. Because the breakout force is so high relative to the force generated at full deflection (almost 50\%), the system damping coefficient could not be computed using the 
amplitudes resulting from free motion after release (i.e., using logarithmic decrement). As a conservative estimate the natural frequency of the joystick was approximated to be the damped frequency.

In a 2017 experiment Bachelder (Ref. 22) identified a nonlinear pilot control technique termed 'amplitude clipping,' whereby the pilot responds to error as predicted by the CM up to a certain stick amplitude and holds that amplitude until the error signal reverses and returns, at which time the pilot resumes active continuous tracking. The amplitude at which the control input is capped can vary over time. As will be described in more detail, pilots were also observed to employ pulse control in this experiment. Figures $8 \mathrm{a}$ and $\mathrm{b}$ show close-ups of examples of amplitude clipping and pulse control, focusing on the regions where the stick moves out of the neutral position. The upward stick rate for amplitude clipping is approximately constant at $180 \% / \mathrm{sec}$, and the stick rate for pulse control is roughly a constant $1,250 \% / \mathrm{sec}$. Assuming that most of the force generated by the joystick is due to spring force, a constant stick rate would result from a constant rate of change of force. Figure $8 \mathrm{~d}$ gives the two force rates for the two control techniques. Further assuming that the force rate computed from the observed stick motion exists prior to stick motion (i.e. before the breakout force is reached, shown again in Figure 8c, the time elapsed from zero force to FB is shown in the last row of Figure 8d.

Amplitude clipping incurs a 0.3 second delay between the first application of stick force and when stick motion initiates, which roughly coincides with the region of quiescence observed prior to motion (for this example). Pulse control would incur only a 0.05 second delay due to FB - this, in conjunction with the gamepad's high natural frequency $(\approx 240 \mathrm{rad} / \mathrm{s})$ would strongly suggest that the gamepad's feel system $Y_{F S}$ can be assumed unity when pulse control is employed. However, when amplitude clipping is used, the relatively high delay due to FB suggests that the effect arising from breakout force should be included in $Y_{F S}$ when amplitude clipping is utilized by the pilot.

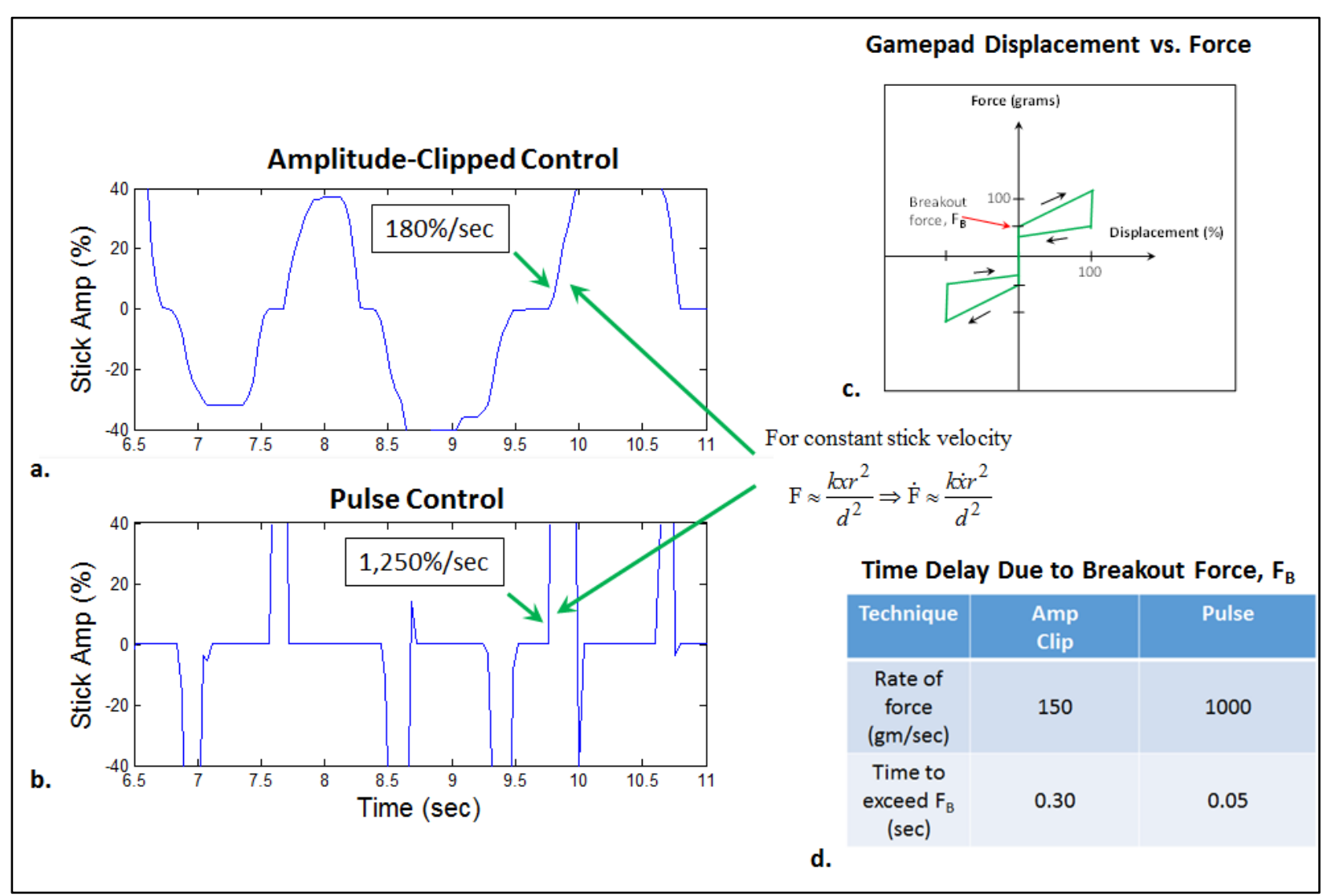

Figure 8. Effect of pilot strategy on time delay due to breakout force: a) Stick history using amplitude clipping; b) Stick history using pulse control; c) Gamepad displacement vs. force; d) Time delay comparison due to breakout force. 


\section{Workload Results}

In this station-keeping experiment the variables that correlated best with subjective rating were stick rate and display error rate. The two variables were combined in an empirically-derived relationship and to produce Bedford estimates of the 92 data points shown in Figure 9a (coefficient of determination is 0.91). Figure $9 \mathrm{~b}$ gives the operations conducted on stick position and display error to produce the Bedford estimate.

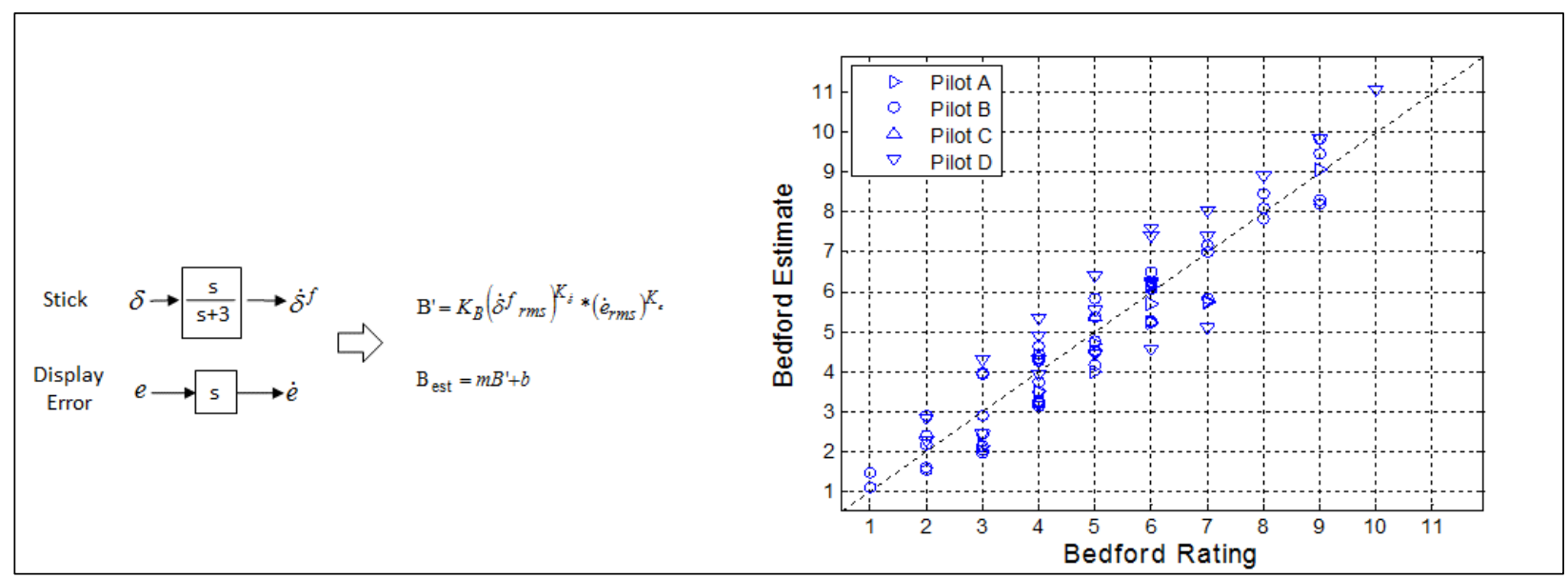

Figure 9. Bedford Estimate: a) Results (coefficient of determination 0.90); b) Computation of Bedford Estimate.

\section{Pulse Model Development}

In the experiment two of the four pilots were observed to use different pulse techniques when controlling the acceleration and jerk command dynamics. Figure 10a shows the pulse width modulation (PWM) technique, where the gamepad joystick was rapidly deflected to the stops and released, so that pulse firing and pulse width were predominantly the only two control variables. In digital control PWM creates a square wave, a signal switched between on and off, to create analog results with digital means. Figure 10b shows the pulse width-amplitude modulation (PWAM) technique (coined by the author), where stick motion consists of pulses for motion that reaches the stops, and amplitude-varying impulses otherwise.

\section{Pulse Width Modulation}

Pilot 1

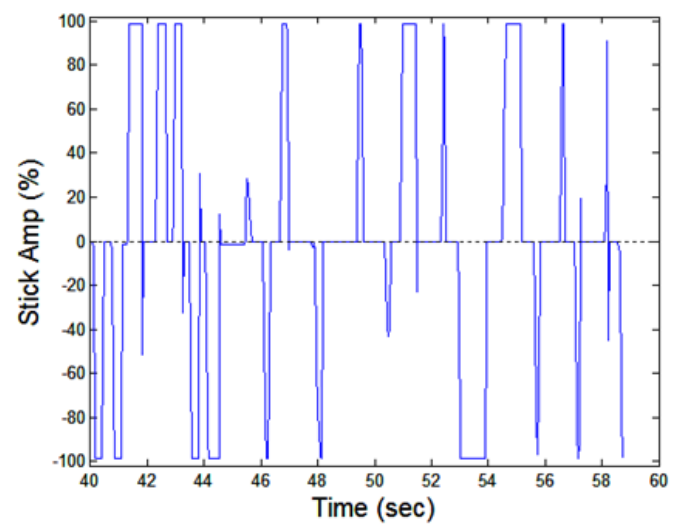

a.

\section{Pulse Width-Amplitude Modulation Pilot 2}

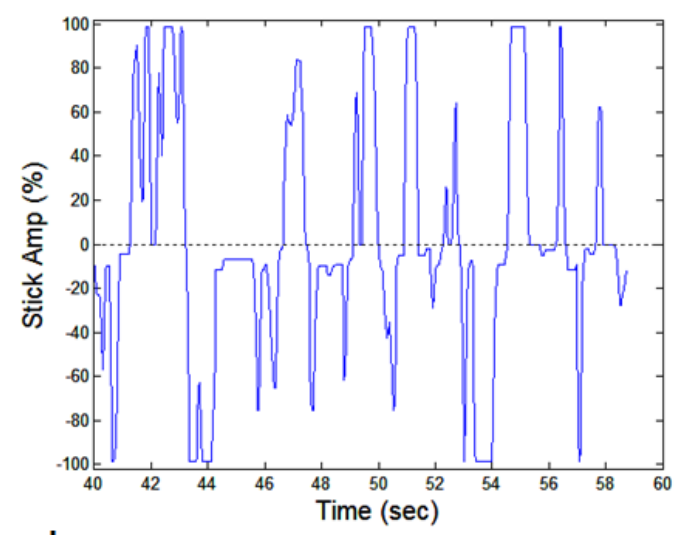

b.

Figure 10. Pilot control techniques used in tracking task. a) Pulse width modulation; b) Pulse widthamplitude modulation.

Model development was conducted using the recorded pilot data (error, stick). The recorded error will be referred to as the open-loop $(\mathrm{OL})$ error $\left(\mathrm{e}_{\mathrm{OL}}\right.$, Figure 11). For robustness, closed-loop (CL) simulation was employed for model 
verification, and the tracking error arising from this is denoted as $\mathrm{e}_{\mathrm{CL}}$. As would be expected, there was a slightly better match between the simulated OL and experimental stick response compared to when CL simulation was used. Since the actual pilot is time-varying, using the recorded (experimental) error to drive the pilot model should produce less mismatch with the output. However, the CL frequency response was observed to produce a better match. This latter result could also be expected since the frequency content of the CL simulated error should have greater linear correlation with the input than what the pilot produced. Finally, there can be surprises related to stability and other factors that are exposed with CL simulation that are absent in the OL, especially when the model makes use of rule-based discontinuities and pulsive control.

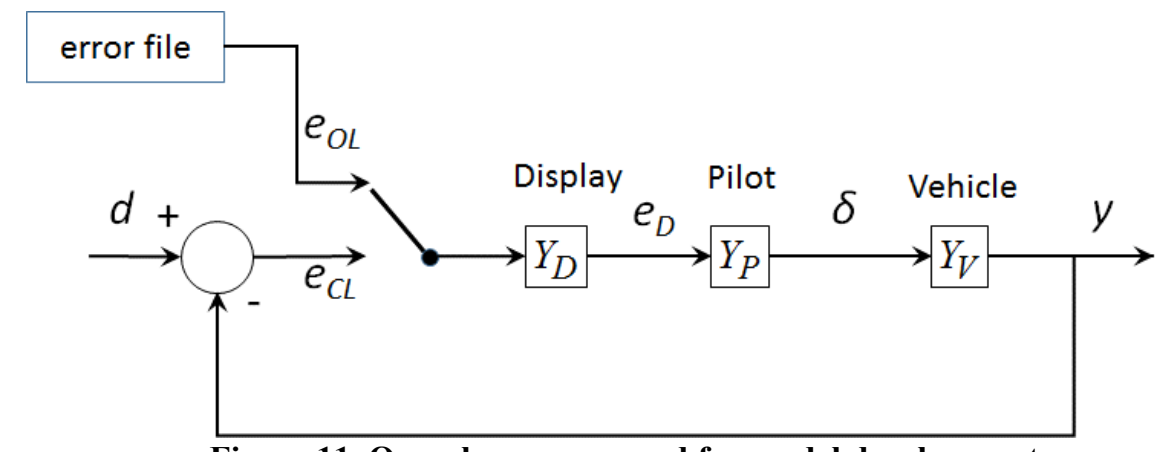

Figure 11. Open-loop error used for model development.

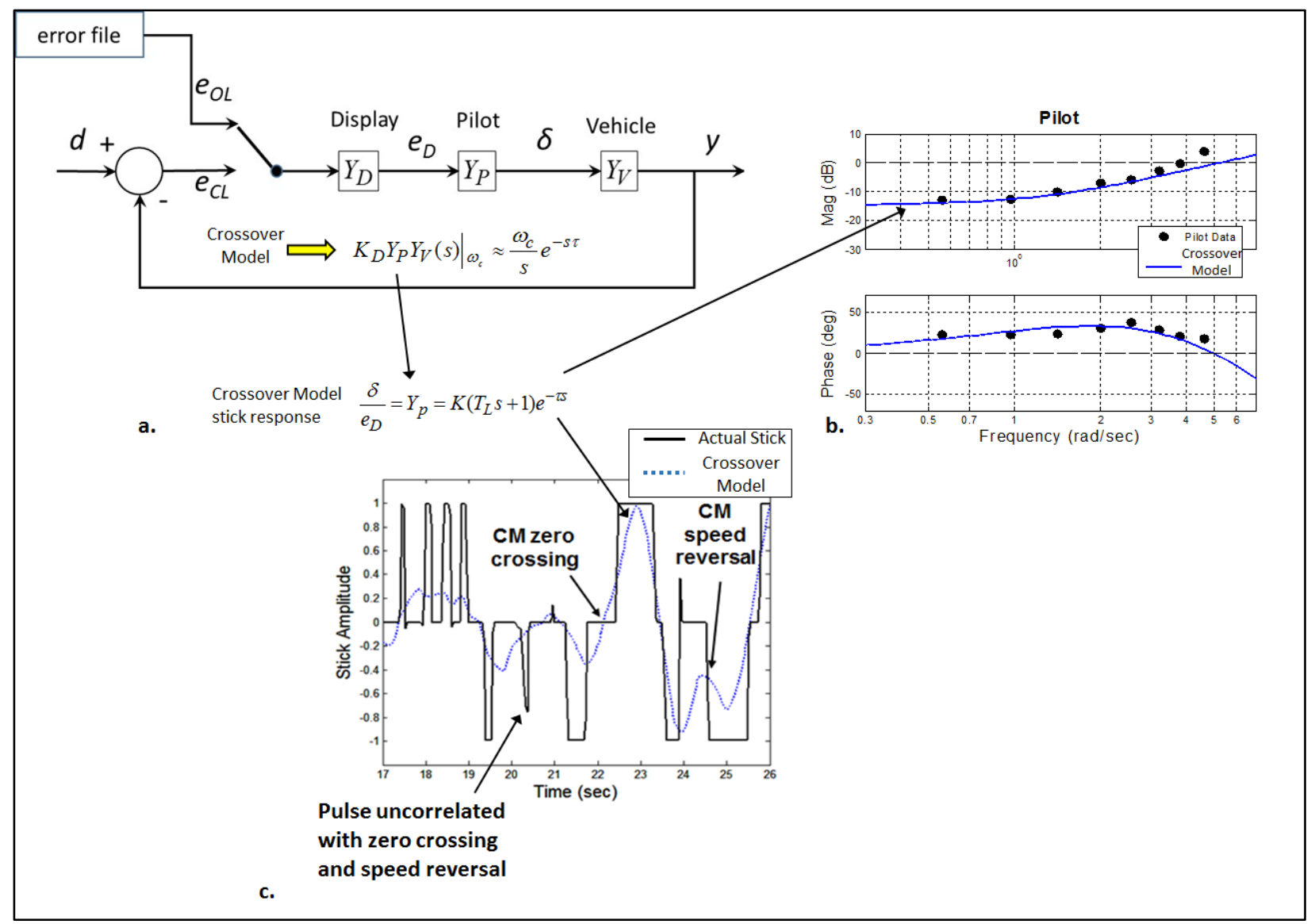

Figure 12. Crossover model employment for PWM logic identification. a) Basic Crossover Model elements; b) Identified pilot frequency response for PWM technique; c) Crossover Model stick signal overlaid on actual stick history showing correspondence between pulsing and Crossover Model's stick signal. 
At the time that the pulse models were developed the $C M$ was used to characterize the pilot element. However, both the Structural Model and the $C M$ yield equivalent frequency responses for the pilot ( $\delta / e$ in Figure 12a) in the region of crossover (Ref. 6). The following describes the procedure that was employed to recognize and formulate the pulse logic for both the PWM and PWAM techniques. Using the actual pilot error, $\mathrm{e}_{\mathrm{OL}}$, the open loop frequency response ( $y / e$ in Figure 12a) was computed, from which the crossover frequency $\omega_{c}$ and the effective time delay $\tau$ were estimated. Assuming the pilot acts like a first-order lead with a time delay (see $Y_{p}$ in Figure 12a), when crossover occurs $K$ can be written in terms of known constants and the unknown lead term $T_{L}$. This is substituted back into $Y_{p}$ so that the only unknown is $T_{L}$, and a best-fit is performed (solid blue line in Figure 12b) with pilot's magnitude and phase (filled circles in Figure 12b) to yield $T_{L}$, hence $Y_{p}$. The output of $Y_{p}$ is termed the $C M$ stick response, and this was computed in time over the run and compared with the actual stick pulses (Figure 12c). It was observed that in general a pulse is triggered by zero-crossings and speed reversals in the $C M$ stick response. Figure $12 \mathrm{c}$ also shows a pulse that does not correlate with either a zero crossing or a speed reversal, and this kind of pulse occurred often enough to warrant examining its trigger source.

In Figure $13 \mathrm{~b}$ the areas under actual and crossover stick signals are integrated in time, with integration starting and ending when the sticks change polarity. It is seen that the integrations approximately match when the stick polarities flip. By inspection pulses were observed to obey the logic shown at top of Figure 13, where a pulse ceases if the area under the actual stick is greater than some fixed percentage of the area under the crossover model's signal. Pulses are triggered when the crossover model's stick encounters a zero crossing or speed reversal pulse, or when the area under the actual stick is less than some fixed percentage of the area under the $C M$ 's signal. It was also observed that there is a generally consistent delay between when a zero crossing or speed reversal occurs, and when a pulse is actually triggered. This appears to be a safeguard against spurious triggering by requiring a certain amount of time to pass and for the amplitude to rise above some minimum threshold before the operator commits to a pulse. The logic governing PWAM was similar to PWM, except that the variable pulse amplitude was assigned to the CM's stick amplitude at the time of pulse trigger multiplied by a constant.

Figure 14 compares the modeled and actual stick outputs for the PWM and PWAM styles, and they subjectively appear to agree well. In the next section, modeled and actual behavior will be compared using frequency response as well as probability distributions of the stick amplitude and periods of stick inactivity (quiescence).

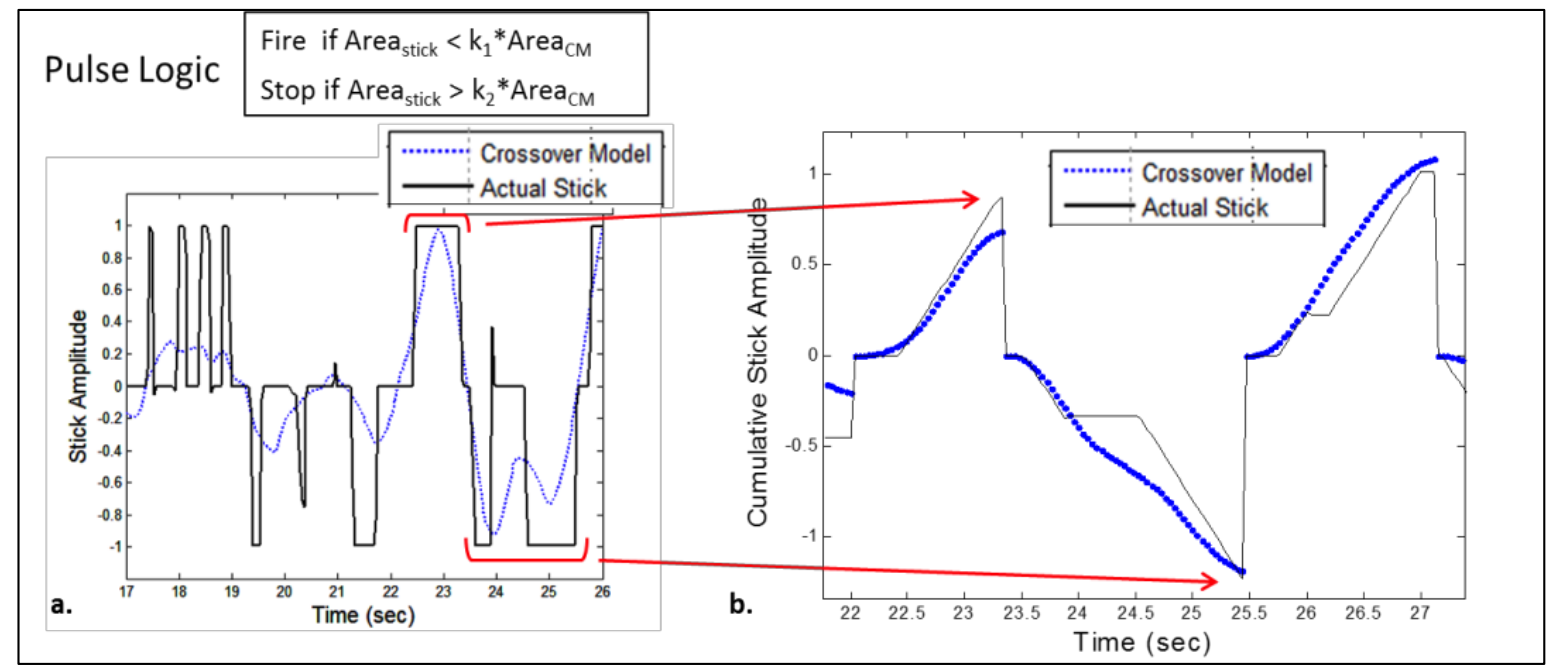

Figure 13. PWM Crossover Model and stick integration. a) Crossover Model stick signal overlaid on actual stick; b) Comparison Crossover Model integration and actual stick integration (they are roughly equal at each zero crossing of the Crossover Model ouput). Pulse logic shown at top, governing pulse width and pulses not associated with zero crossing and speed reversal. 


\section{Pulse Width Modulation Pilot 1}

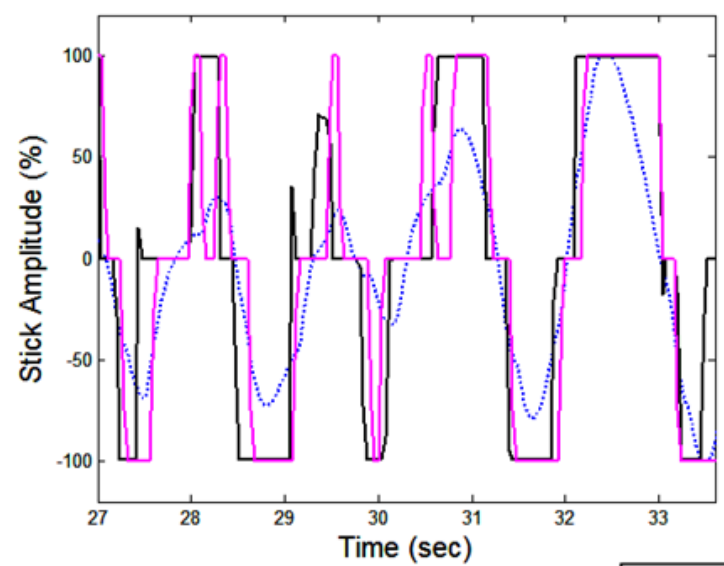

Pulse Width-Amplitude Modulation Pilot 2

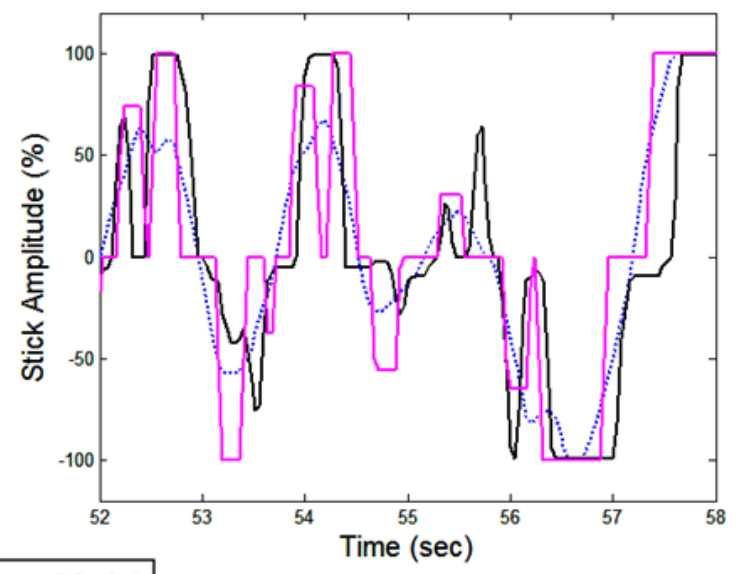

Crossover Model

Actual Stick

Pulse Model

Figure 14. Comparison of pulse models with actual data: a) Pulse Width Modulation; b) Pulse Width Amplitude Modulation.

\section{Model Verification}

Examples are now given where the Structural Model is used to identify the pilot element for the PWM, PWAM, and amplitude clipping control techniques. The pulse-like motion observed for PWM and PWAM (as was shown in Figure 10) is generally characterized as rapid motion until the joystick stops are hit and held a finite time before stick release, or the stick reaches some maximum amplitude and is then released. Such rapid and uniform rate of motion implies precognitive control, where the muscles follow a 'canned' procedure that is only modified to reach the terminus. This would contrast with the continuous tracking control described by McRuer's neuromuscular model in Reference 13, where a relatively constant mean tension is maintained by the an agonistic/antagonistic limb muscle pair. For motion to occur, one muscle of the pair must generate a force greater than that of the other, and thus the tension levels must fluctuate about the average. This model and its operating frequencies would not permit the impulsive, sharp-edged stick response observed in pulse control, thus as a first approximation the neuromuscular element $Y_{N M}$ is assumed to be unity when modeling pulse control.

When amplitude-clipping control is modeled, rather than iterate on the undamped natural frequency of the open loop neuromuscular system $\left(\omega_{N M}\right)$ along with the other parameters of the Structural Model (Figure 3) until a best-fit with the observed data is obtained (or fixing $\omega_{N M}$ at some assumed value), the power spectrum of pilot's stick was examined. Figure 15a shows the power spectrum density (PSD) of the stick when amplitude clipping was used to control acceleration command dynamics. The frequencies of the sum-of-sines forcing function are denoted with open circles, the highest frequency located approximately at $5 \mathrm{rad} / \mathrm{sec}$. Figure $15 \mathrm{~b}$ is a close-up beyond $5 \mathrm{rad} / \mathrm{sec}$, and power can be observed up to about $10 \mathrm{rad} / \mathrm{sec}$, with a concentration at around $7 \mathrm{rad} / \mathrm{sec}$. When jerk-command dynamics are controlled (again using amplitude clipping), Figure 15d shows the power to be more evenly distributed between 5 and $10 \mathrm{rad} / \mathrm{sec}$. Based on these observations, $\omega_{N M}$ was fixed at $8 \mathrm{rad} / \mathrm{sec}$ when amplitude clipping was used as the control style. This is lower than the value used by Hess in Ref. 7, which is likely due to the different inceptor and limb characteristics (the gamepad joystick is controlled by the thumb). 

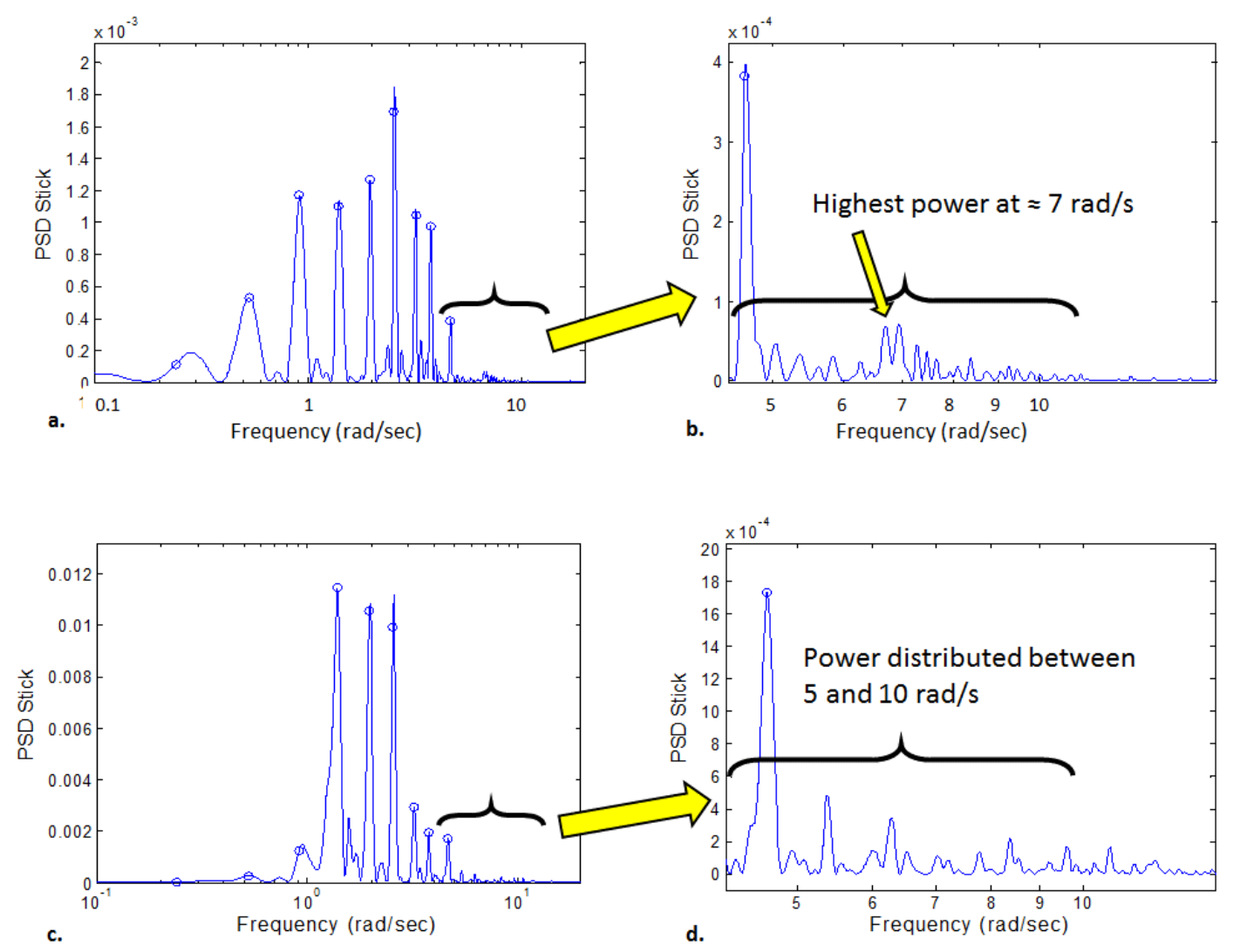

Figure 15. Spectral decomposition of stick response . a) Using acceleration-command vehicle dynamics (forcing function power denoted by open circles); b) Close-up beyond forcing function power . c) Using jerkcommand vehicle dynamics; b) Close-up beyond forcing function power .

From Ref. 7 the pilot time delay was fixed at 0.20 seconds, and the five other parameters of the pilot Structural Model $\left(K_{e}, T_{L}, K_{\delta}, T_{K}, \varsigma_{N M}\right)$ were iterated in a Simulink model of Figure 3 to minimize the cost function $J$ given in Eqn. 2.

$$
J=\gamma_{1} * \sum_{i=1}^{8} \Delta m a g_{i}{ }^{Y P V}+\gamma_{2} * \sum_{i=1}^{8} \Delta p h s_{i}{ }^{Y Y v}+\gamma_{1} * \sum_{i=1}^{8} \Delta m a g_{i}{ }^{Y p}+\gamma_{2} * \sum_{i=1}^{8} \Delta p h s_{i}^{Y p}+\gamma_{3} * \Delta \sigma(\delta)+\gamma_{4} * \Delta P D F
$$

In Eqn $2 \Delta\left(\right.$ ) refers to the absolute difference between observed and simulated variable ( ). $\Delta \operatorname{mag}_{i}{ }^{Y p Y v}$ thus denotes the difference in the open loop magnitudes $(Y p Y v$, in $\mathrm{dB}$ ) between observed and simulated at each frequency $i$ of the forcing function, $\Delta p h s_{i} Y p Y v$ denotes the difference in the open loop phase, and $\Delta m a g_{i}{ }_{i}$ and $\Delta p h s_{i}{ }^{Y p}$ use the magnitude and phase of the pilot element, respectively. $\sigma(\delta)$ is the standard deviation of the stick, and $P D F$ is the probability density function of the stick amplitude (see Figure 20a). The elements in Eqn 2 are weighted by constants $\gamma$. The time domain metrics $(\sigma$ and $P D F)$ were included since it is possible for a very different stick distribution and $\sigma$ to produce the same frequency response. The parameters minimizing the cost function for four simulation runs are given in Table 2, along with various metrics. The estimated Bedford rating (computed from the modeled response as shown in Figure 9), $B_{\text {est }}$, and the actual Bedford rating are given in Table 2. Also compared is the relative correlated output $\rho^{2}$, defined as the portion of the power in the stick output which exists at the disturbance frequencies, divided by the total stick power. For instance, in Figure 15a, summing the areas flanking each forcing frequency (out to the first local minimum in PSD) yields the correlated power, and this is divided by the total PSD area. 


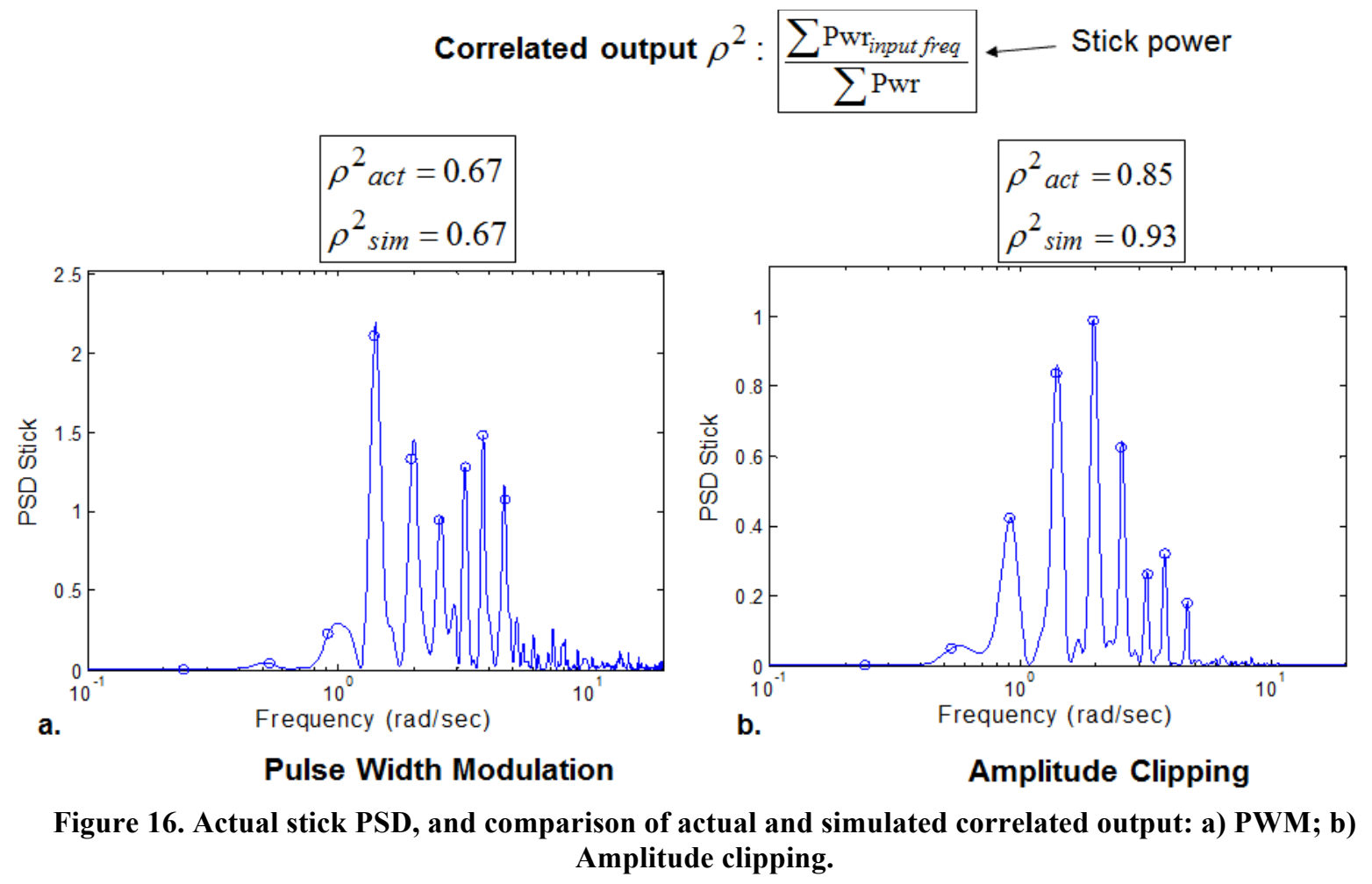

As discussed earlier, closed-loop simulation was chosen to conduct model verification (model devleopment employed open-loop simulation), since this would expose any stability problems associated with the model that may have been absent using eOL (the pilot's recorded tracking error, Figure 17.

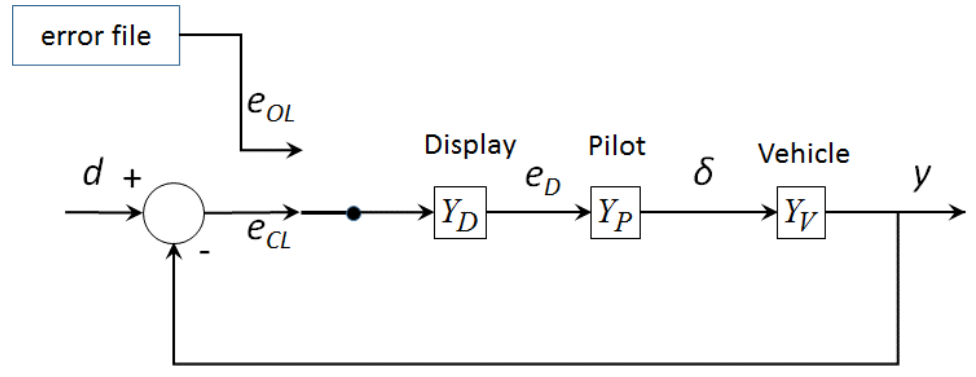

Figure 17. Closed-loop error used for model verification.

Figure 18 shows the identified open loop and pilot frequency responses (computed using cross spectral densities, Ref. 3) from the observed pilot data and from the data generated by the nonlinear simulation (nonlinear due to the pilot technique element $Y_{T E C H}$ ). The solid blue line denotes the linear frequency response produced without the nonlinear pilot technique element. The nonlinear effects of clipping are minimal except at the lower frequencies, where magnitude is reduced. Looking at Table 2 the relative correlated output $\rho^{2}$ for the simulation is 0.93 , also reflecting the technique's minimal impact on remnant. Note that the actual value of $\rho^{2}$ was 0.85 , suggesting that the pilot's contribution to remnant due to internally generated noise is approximately $8 \%(0.93-0.85)$ of the total stick power. Absent an accurate model of pilot technique, the $15 \%$ total remnant observed from the pilot data could not have been segmented into technique and internal noise categories. Figure 19 shows good correspondence between the time histories of the actual and simulated stick amplitudes. Actual and simulated stick probability density functions compare favorably in Figure 20a, as does the distribution of quiescence time in Figure 20b. Quiescence was defined as when the stick's rate fell below a threshold, when stick amplitudes were non-zero 
(i.e. time did not accrue towards quiescence when the stick was in the zero-force, zero-amplitude position). The metrics for this run in Table $2\left(\mathrm{~K} / \mathrm{s}^{2}\right.$ dynamics, AC denotes amplitude clipping), in combination with Figure 18 Figure 20, indicate that the integrated components of the model reproduced the pilot's response with high accuracy. Of note is that the pilot did not employ the kinesthetic feedback loop ( $K_{\delta}$ was zero), relying entirely on equalizing the error channel with gain and lead.
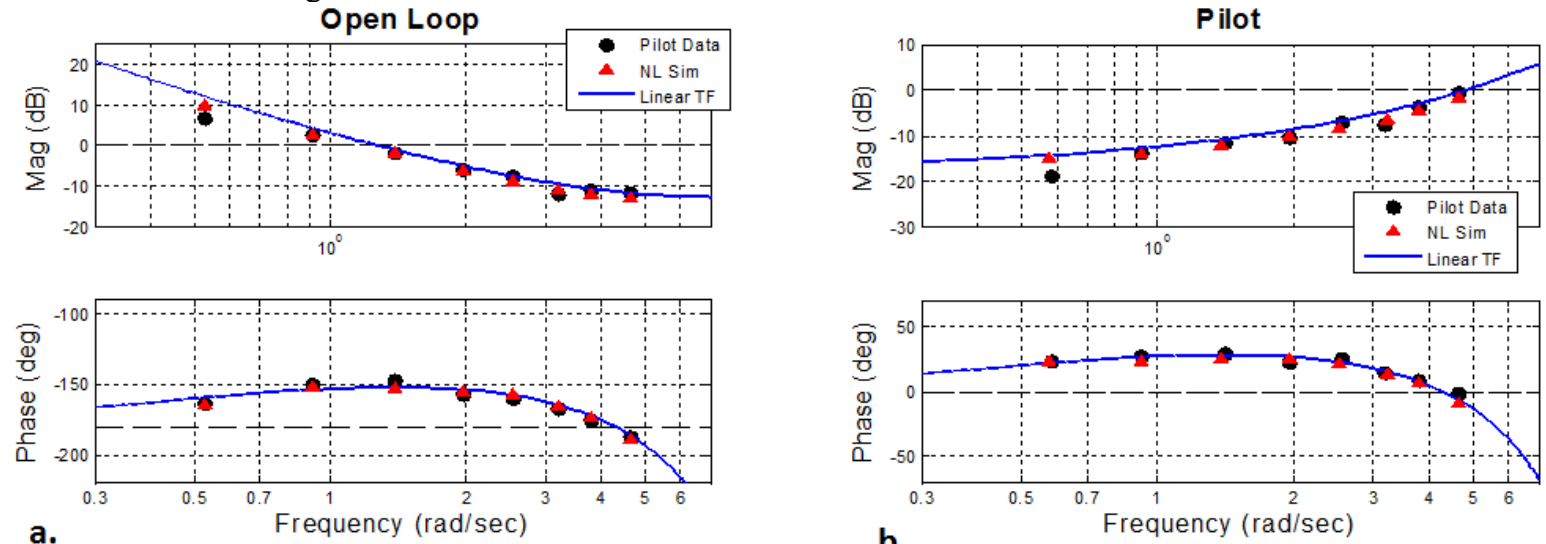

Figure 18. Identified open loop and pilot frequency responses for the pilot data and nonlinear model data, with the linear model overlaid (acceleration-command dynamics, Amplitude-Clipping model).
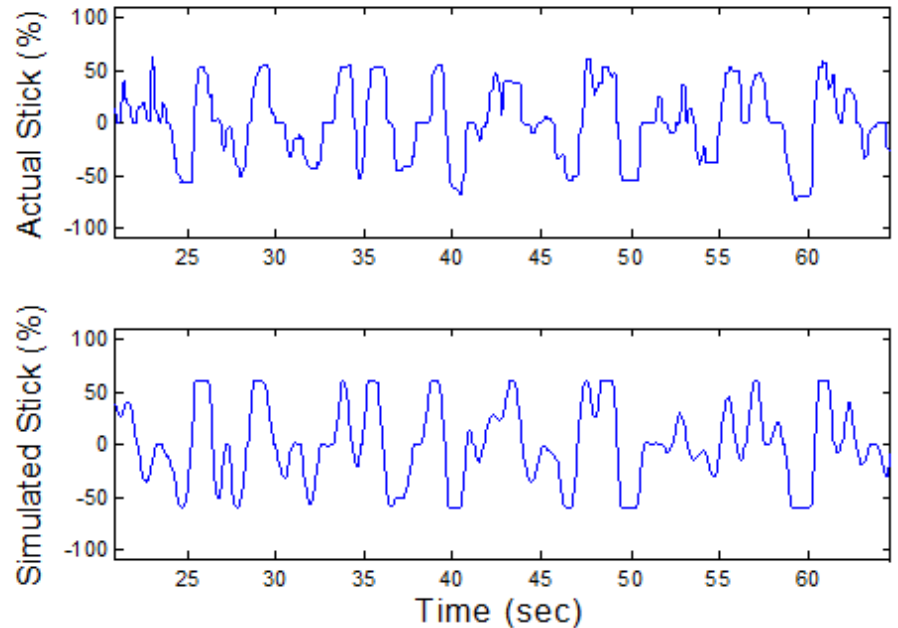

Figure 19. Comparison of actual and simulated stick (acceleration-command dynamics, Amplitude-Clipping model).
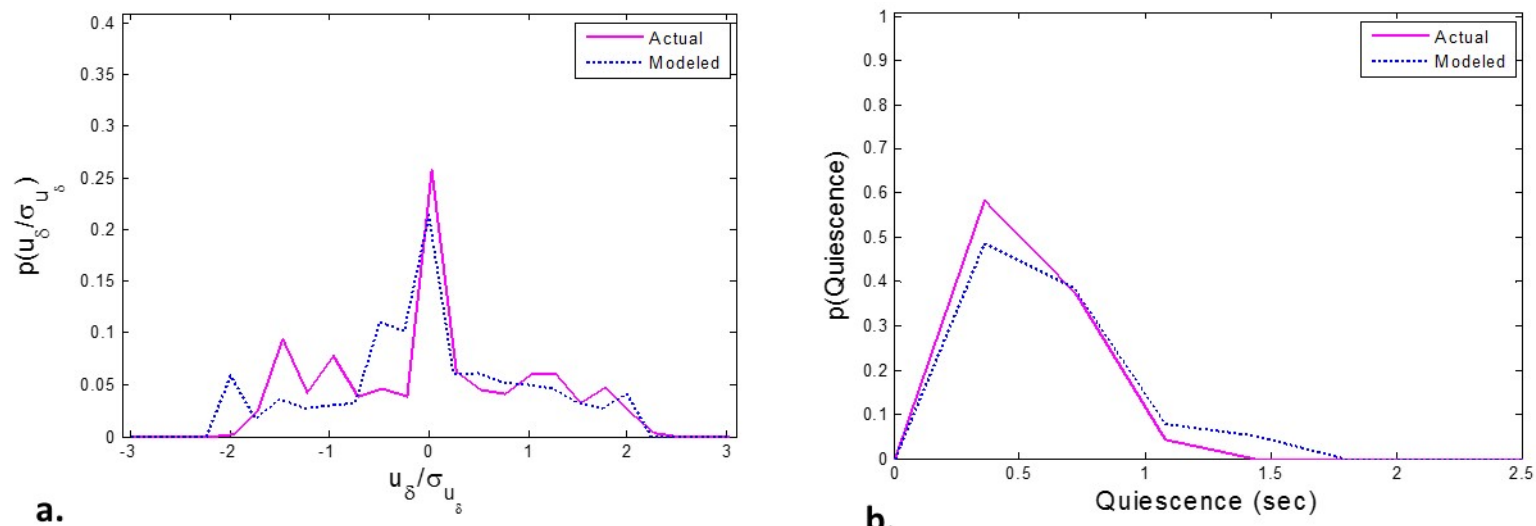

Figure 20. Distributions for stick associated with Figure 19 comparing actual and model: a) Stick amplitude distribution; b) Stick quiescence distribution.

American Institute of Aeronautics and Astronautics 
Simulation of the control style PWAM in the Structural Model is now examined. The sharp edges and corners observed in the stick response for both PWM and PWAM control infer that their neuromuscular dynamics affected frequencies far higher than what was measured when amplitude clipping (pseudo-continuous) control is used. The neuromuscular pilot element $Y_{N M}$ was thus set to unity when modeling PWM and PWAM, eliminating $\omega_{N M}$ and $\zeta_{N M}$ from the identification process. Figure 21 shows very good model agreement with the actual frequency and time responses. The linear response (i.e. if the pilot technique element was left out) in Figure 21b illustrates how highfidelity modeling can expose observed measurements as artifacts of technique rather than the result of internal processing. Ref. 3 describes the phenomenon of low-frequency 'droop' observed in pilots, which was not accounted for by the basic $C M$. This low-frequency phase loss increased with the order of the system being controlled, and was attributed to and reproduced using the inner kinesthetic feedback loop (in Ref. 6). However, in this run the marked difference in phase (and magnitude) between the linear response and the nonlinear response at the lowest frequency is due entirely due to the PWAM technique - if the kinesthetic feedback were contributing to the phase and magnitude droop this would have caused the linear response to tend toward the modeled nonlinear response. The actual measured phase agrees very well with the nonlinear model, including at the higher frequencies where the pilot phase also departs from the linear response. Phase droop also occurs with rate and position command dynamics (Ref. 3), where continuous control would certainly be employed and technique would not be a factor, but this example highlights the potential importance of including pilot technique when assigning causality.

Looking at Table 2, the pilot made substantial use of inner-loop proprioceptive feedback $\left(K_{\delta}=0.25\right)$, and remnant due to technique $\left(1-\rho_{\text {sim }}^{2}=24 \%\right)$ was considerably larger than when amplitude clipping was employed by the other pilot (where 1- $\rho_{\text {sim }}^{2}=7 \%$ ). Despite the seemingly stochastic nature of PWAM, the pilot's internal remnant (4\%) was half the internal noise associated with the amplitude clipping technique. This is quite remarkable given that the pilot appears to be simultaneously integrating both the physical stick position as well as the internallygenerated stick position to generate the pulse commands.
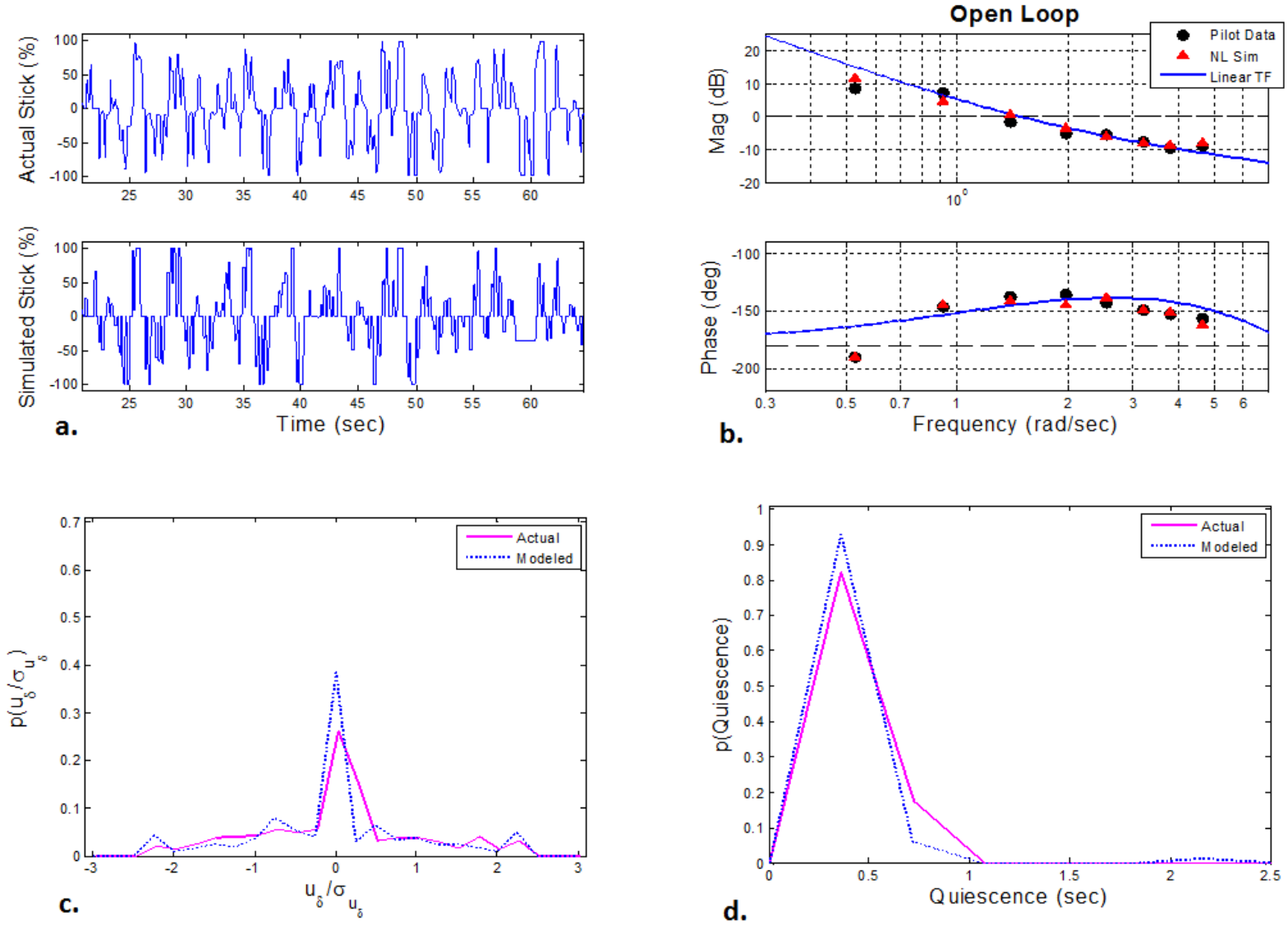

Figure 21. Pulse Width Amplitude Modulation (acceleration command dynamics): a) Comparison of actual and simulated stick; b) Frequency response comparision of open-loop; c) Stick amplitude distribution; d) Stick quiescence distribution. 
The frequency and time domain results in Figure 22 show excellent correspondence between modeled and pilot data for PWM control. It is curious that the PWM control technique produced 33\% remnant, and since this coincided with the remant observed in the experimental data then the pilot apparently generated almost no internal noise (given the model's accuracy). The technique also appears to be responsible for the leveling of magnitude at the higher frequencies (the observed and nonlinear model magnitudes match, departing from the linear response). Kinesthetic feedback was activated $\left(K_{\delta}=0.10\right)$, but to a lesser extent than with PWAM.
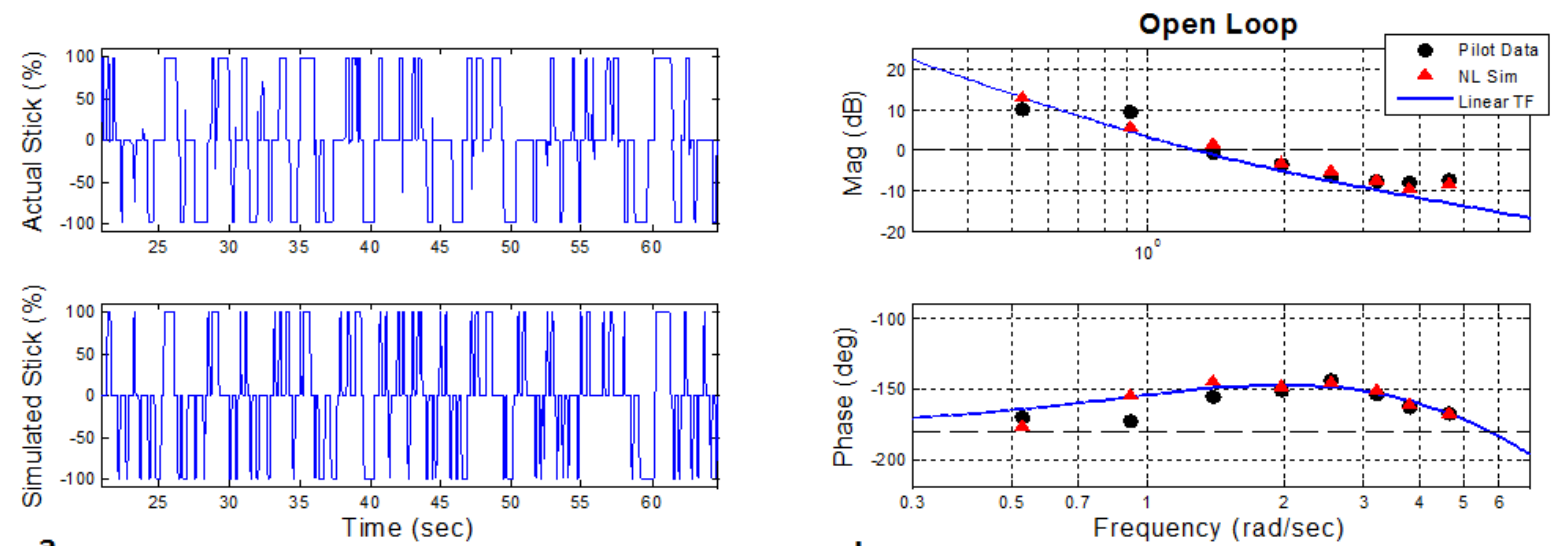

b.
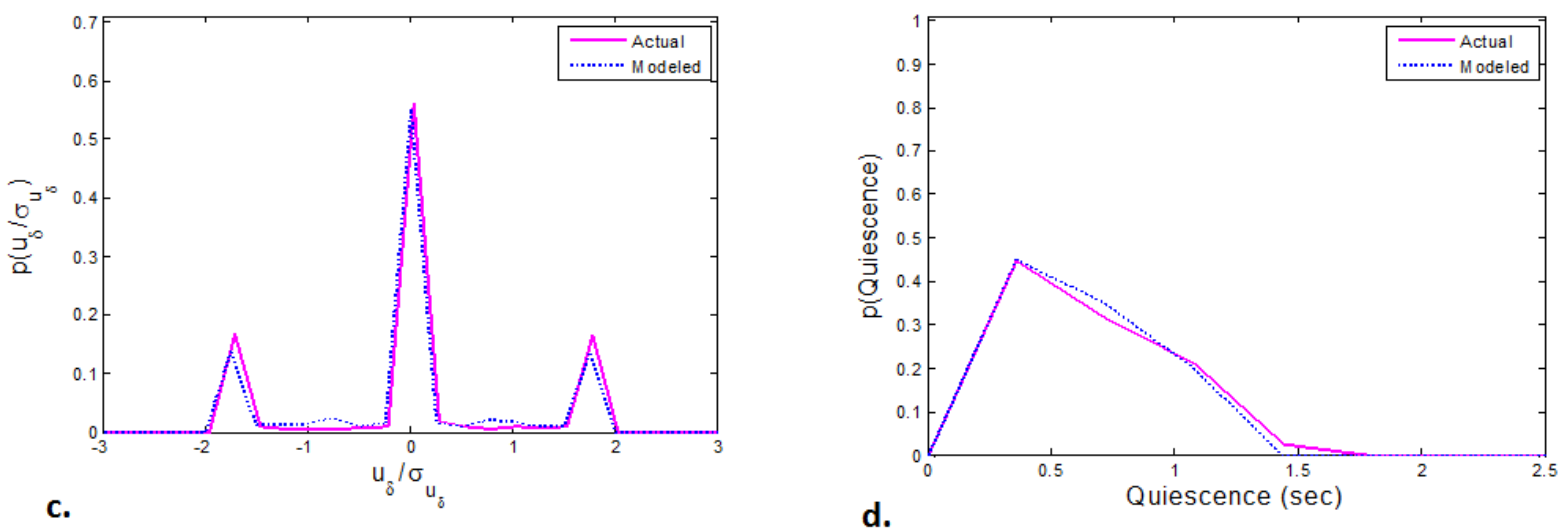

Figure 22. Pulse Width Modulation (acceleration command dynamics): a) Comparison of actual and simulated stick; b) Frequency response comparision of open-loop; c) Stick amplitude distribution; d) Stick quiescence distribution.

The last example examines a run that used amplitude clipping to control jerk dynamics, which are extremely challenging to stabilize and to conduct tracking with. In Figure 23 the model produced a good match with the pilot data in frequency response and stick amplitude distribution, and a fair match in the time response and quiescence distribution. The internal noise (remnant) of the pilot is the highest (11\%) out of the four runs investigated, and in contrast to when this pilot was controlling acceleration command dynamics, he now employed proprioceptive feedback $\left(K_{\delta}=0.10\right)$ and used the largest lag time constant $\left(T_{K}=1.13\right.$, more than double what the other two pilots operated at). 

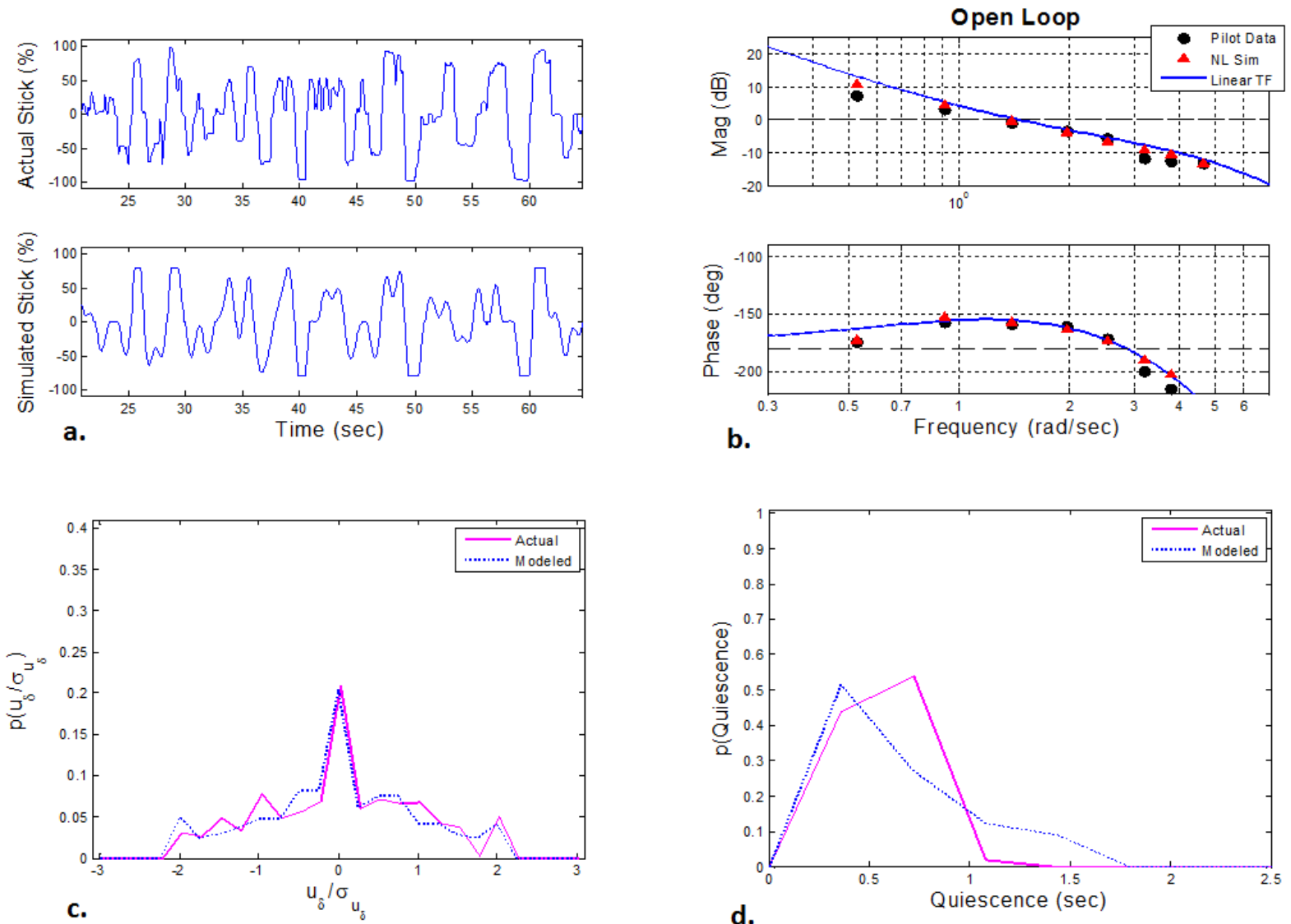

Figure 23. Amplitude Clipping (jerk command dynamics): a) Comparison of actual and simulated stick; b)

Frequency response comparision of open-loop; c) Stick amplitude distribution; d) Stick quiescence distribution.

Table 2. Pilot model parameter values used to generate describing functions, and comparison of simulated and actual measures.

\begin{tabular}{|c|c|c|c|c|c|c|c|c|c|c|c|c|c|c|c|c|}
\hline $\begin{array}{l}\text { Controlled- } \\
\text { element } \\
\text { dynamics }\end{array}$ & $\begin{array}{l}\text { Control } \\
\text { technique }\end{array}$ & $\mathrm{K}_{\mathrm{e}}$ & $\mathrm{T}_{\mathrm{L}}$ & $\mathrm{K}_{\delta}$ & $\mathrm{T}_{\mathrm{K}}$ & $\tau$ & $\zeta_{n}$ & $\omega_{n}$ & $\sigma\left(\delta_{\operatorname{sim}}\right)$ & $\sigma\left(\delta_{\mathrm{act}}\right)$ & $\sigma\left(e_{\text {sim }}\right)$ & $\sigma\left(e_{a c t}\right)$ & $\rho^{2}{ }_{\operatorname{sim}}$ & $\rho_{\text {act }}^{2}$ & $B_{\text {est }}$ & $\mathrm{B}_{\text {act }}$ \\
\hline $\mathrm{K} / \mathrm{s}^{2}$ & $A C$ & 0.19 & 0.96 & 0 & - & 0.20 & 0.3 & 8 & 0.32 & 0.33 & 1.00 & 1.10 & 0.93 & 0.85 & 6.4 & 6 \\
\hline $\mathrm{K} / \mathrm{s}^{2}$ & PWAM & 0.40 & 0.60 & 0.25 & 0.43 & 0.20 & 0.3 & 8 & 0.43 & 0.43 & 0.86 & 0.95 & 0.76 & 0.72 & 6.2 & 6 \\
\hline $\mathrm{K} / \mathrm{s}^{2}$ & PWM & 0.25 & 0.80 & 0.10 & 0.48 & 0.20 & 0.3 & 8 & 0.60 & 0.60 & 0.87 & 1.10 & 0.67 & 0.67 & 6.8 & 6 \\
\hline$K / s^{2}(s+3)$ & $A C$ & 0.35 & 0.55 & 0.10 & 1.13 & 0.20 & 0.3 & 8 & 0.43 & 0.47 & 1.36 & 1.84 & 0.94 & 0.83 & 8.5 & 8 \\
\hline
\end{tabular}

With the evidence suggesting that both PWM and PWAM techniques integrate the internal stick signal and the physical stick position, the Structural Model has been modified in

Figure 24 with technique-dependent switches that can enable the integration paths to the pilot technique element when needed. Since the two pulsive techniques required pure kinesthetic integration of the stick, it seems reasonable to assume that this same information would be leveraged by the first-order lag in the kinesthetic feedback loop (see YPF in Figure 3). Coincidentally PWM and PWAM were both observed to employ this loop. 


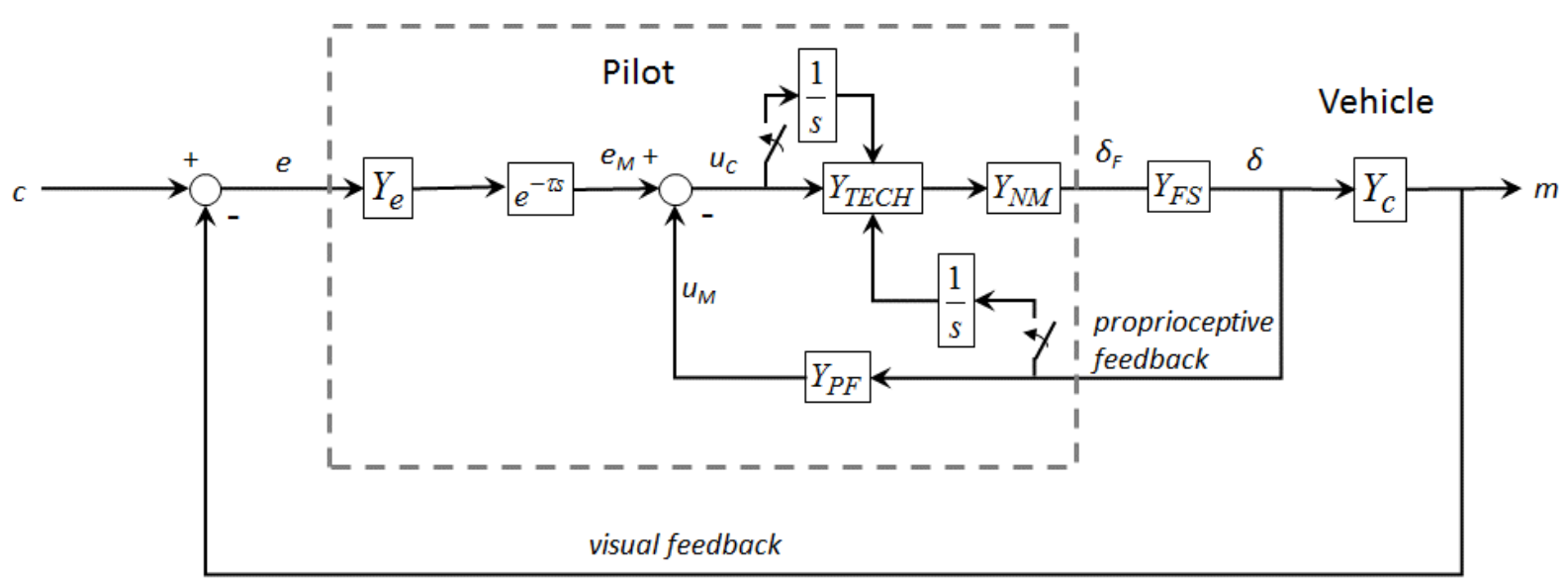

Figure 24. Pilot Structural Model showing integration of internal stick and physical stick signals as technique-dependent inputs to the pilot technique element.

\section{Pilot Cost Index Development}

Looking at Table 2, the matrix of cause and effect is rich, complex, and convoluted. In general, the higher the time constant that the pilot must generate the more difficult ( $T_{L}$ is the lead time constant in the error path, $T_{K}$ is the lag time constant in the proprioceptive path). The degree that these are employed is governed by the gains operating on those paths (respectively, $K_{e}$ and $K_{\delta}$ ). The relative use of the paths is indicated by the ratio $K_{e} / K_{\delta}$. Tracking error and workload appear to be affected by vehicle dynamics, pilot technique appears to influence workload and tracking, and parameter selection may be influenced by - and influence - a host of variables. Past attempts at assigning a rating or a rating increment to individual parameter values or a ratio of parameters met with limited success (Ref. 3, Ref. 23). An operator will probably never vary just one parameter and while the others are unchanged - if one varies then the others will likely shift as well. For a given operational configuration (technique, skill level, display, vehicle, environment, etc.), the pilot should tend toward a set of internal settings that minimizes some cost index. This implies that a piecemeal approach is unsuitable - if the vehicle dynamics are altered, (or the display or control gains), the pilot adopts a different set of parameter values, and they shift in concert. The questions posed in this work are: how do they shift, and why?

If a pilot were solely concerned with minimizing workload, no control motion would result. Conversely, if the pilot were purely driven by performance, workload could be extreme. Rather, some balance of the two is probably used. Bachelder (Ref. 22) demonstrated that the product of estimated Bedford workload and tracking error was a cost metric that showed potential for predicting - through cost minimization - the gain and lead time constant that a pilot will operate at, for a given task (disturbance, vehicle dynamics, display sensitivity, control technique). By iterating on the pilot model parameters in a simulation of the pilot and vehicle, tracking error and workload were simulated so that parameters could be plotted against cost metric and a minima identified. Similarly in this work, estimates of workload (Bedford) and tracking error were generated using the pilot models, and the product of the two was used to define a pilot cost index (PCI) in Eqn 3.

$$
P C I=B_{e s t} * \sigma(e)
$$


The variation of PCI with the parameters chosen by the pilot is shown in Figure 25. Two of the three equalization parameters selected by the pilot, $K_{e}$ and $T_{L}$, roughly coincided with where PCI was minimized for these parameters, but the location of the proprioceptive feedback gain $K_{\delta}$ used by the pilot $\left(K_{\delta}=0\right)$ differed greatly from the PCI minimum. Since kinesthetic feedback was not employed, the lag time constant $T_{K}$ was irrelevant.
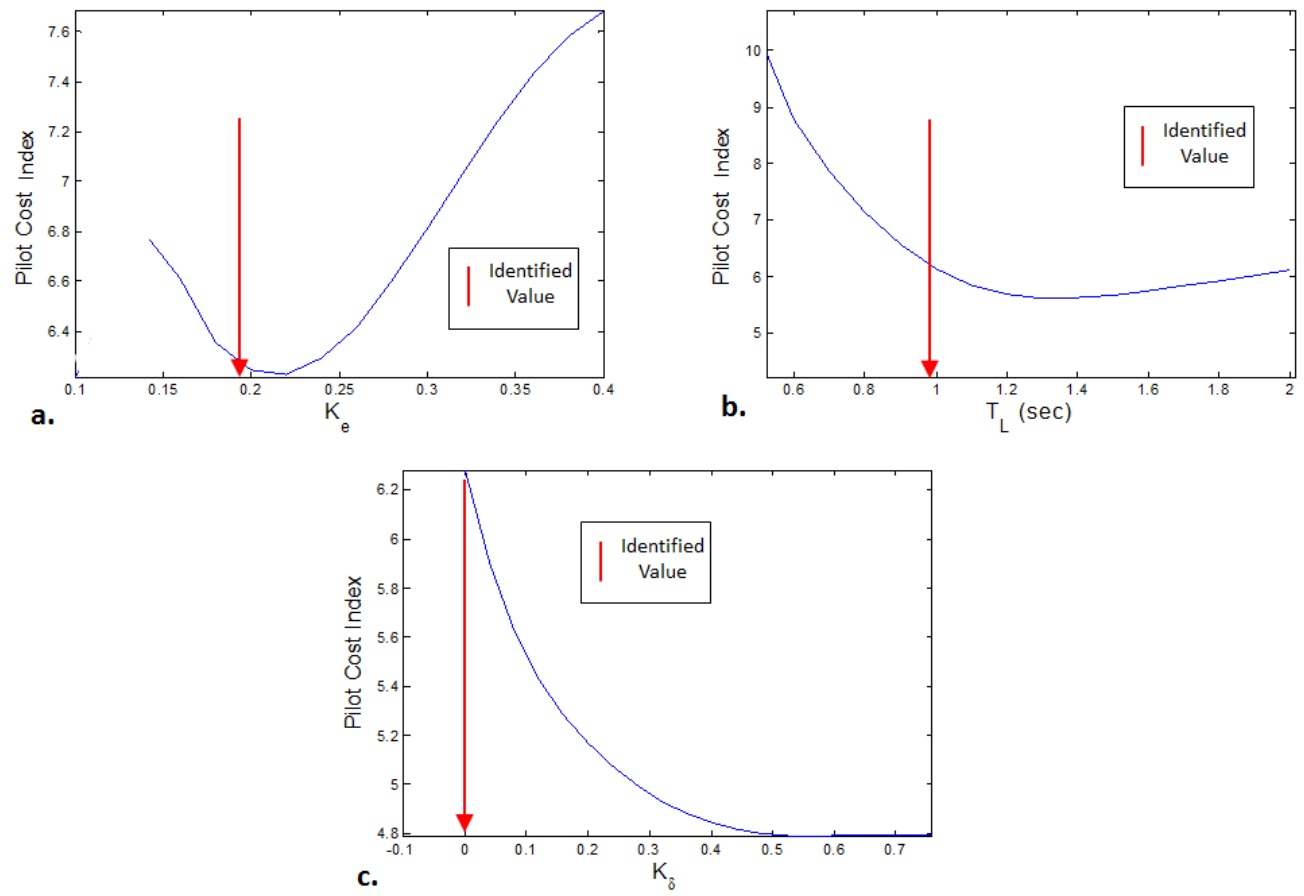

Figure 25. Variation of Pilot Cost Index (without $K_{\delta}$ compensation) with pilot parameters (acceleration command dynamics, Amplitude Clipping): a) $K_{e}$; b) $T_{L}$; c) $K_{\delta}$.
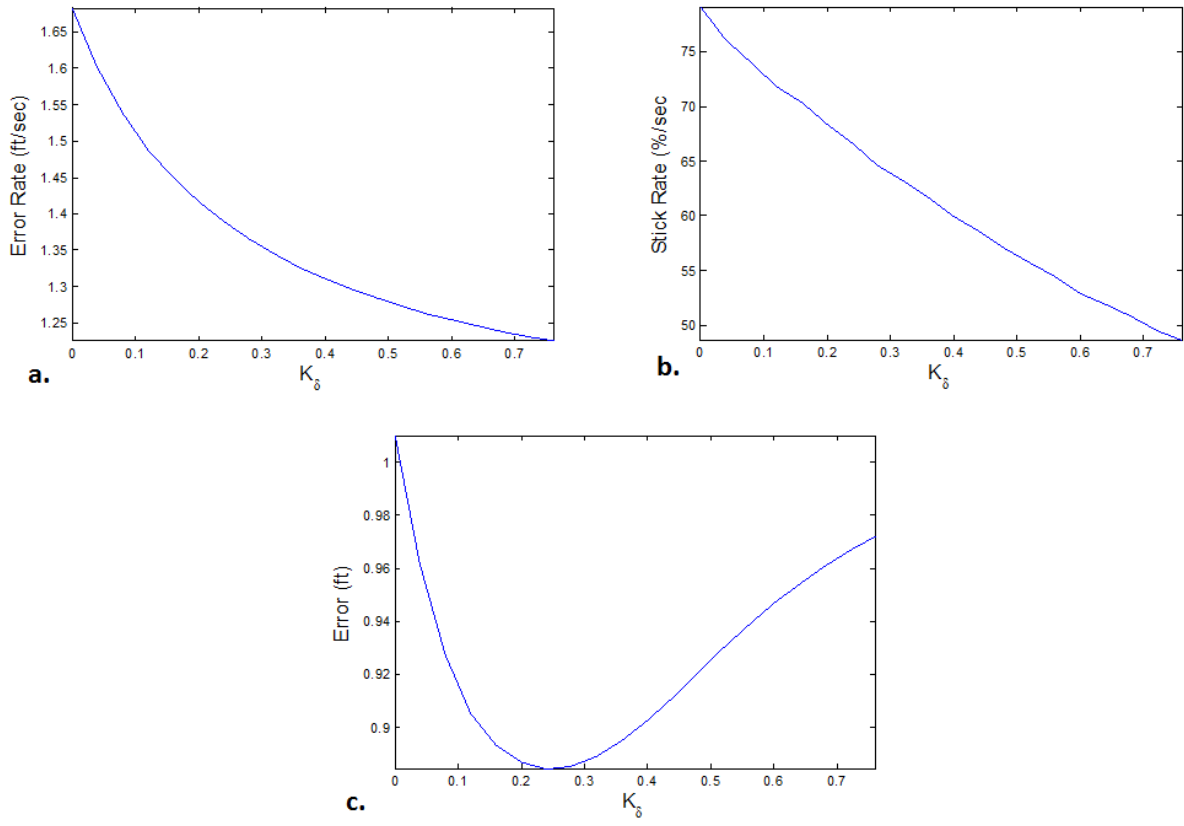

Figure 26. Effect of $K_{\delta}$ on: a) Error rate; b) Stick rate; c) Error.

From a servomechanical perspective, utilizing $K_{\delta}$ would have provided strong advantages, as shown in Figure 26. Error rate and stick rate decrease monotonically with increasing $K_{\delta}$, and position error (Figure 26c) reaches a minimum at $K_{\delta}=0.25$. Despite these benefits, the pilot chose (ableit subliminally) to avoid employing $K_{\delta}$ altogether. Thus it seems there may exist an aversion for the pilot to activate kinesthetic feedback, probably due to the higher- 
level activity in the central nervous system that is required to perform integration, as earlier noted in the section "Structural Model of the Human Pilot."

The contribution of $K_{\delta}$ to PCI was approximated as additive, with $K_{\delta}$ multiplied by a factor $\eta$ as shown in Eqn. 4. The value of $\eta$ selected was such that the revised PCI was minimized when $K_{\delta}=0$. Figure 27a shows the variation of the original PCI with $K_{\delta}$, and Figure 27b shows the desired result using the revised PCI.

$$
P C I=B_{e s t} * \sigma(e)+\eta * K_{\delta}
$$

The effect of the revised PCI does not shift the locations of $K_{e}$ and $T_{L}$ corresponding to the minima shown in Figure 25 , since the entire PCI curve in each case is shifted upward by the same amount $\eta^{*} K_{\delta}$.
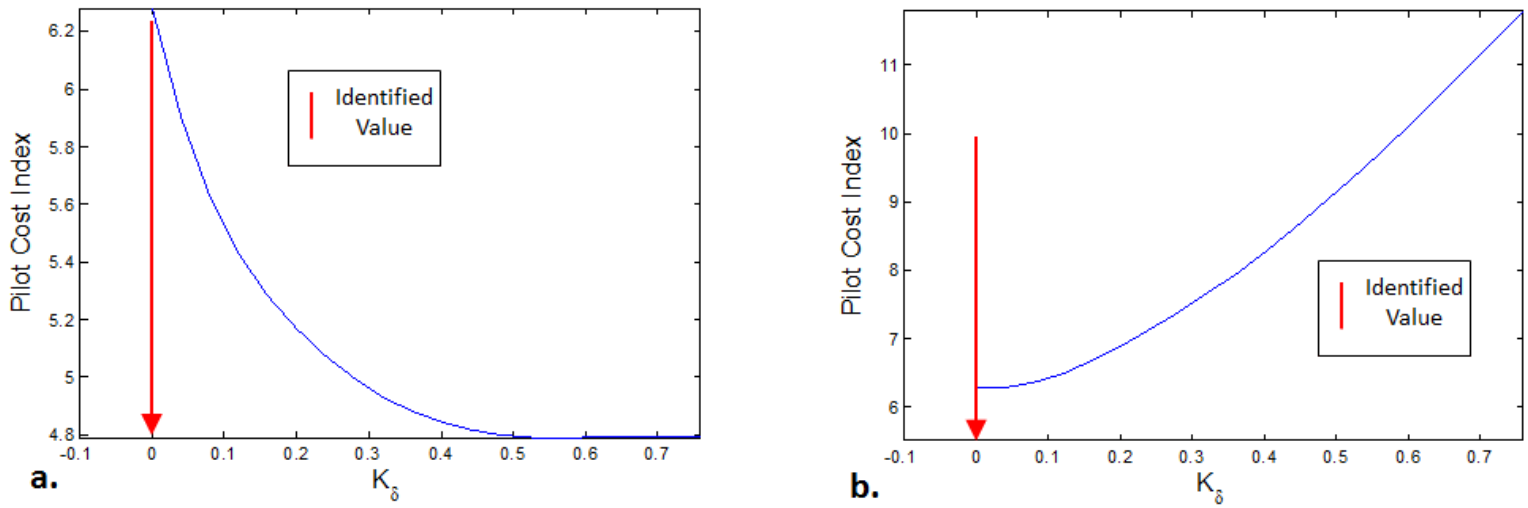

Figure 27. Variation of Pilot Cost Index with proprioceptive feedback gain $K_{\delta}$ (acceleration command dynamics, Amplitude Clipping): a) No $K_{\delta}$ compensation (original PCI); b) With $K_{\delta}$ compensation (revised PCI).

It will be recalled that the pilot in the example above (acceleration command dynamics) eschewing kinesthetic feedback was seen to employ the mechanism when controlling jerk command dynamics. Thus it is of interest to compare the effect of $K_{\delta}$ inclusion with PCI for the jerk command case. Figure 28a gives the variation of the original PCI with $K_{\delta}$, and what is most striking is: 1) the extremely large values PCI assumes when $K_{\delta}<0.1$, and 2) the precipitous drop in PCI when $K_{\delta}$ approaches 0.1. The actual value selected by the pilot for this run was, coincidentally, 0.1. Beyond this value, PCI in Figure 28a gradually descends to a minimum at $K_{\delta}=0.4$. Using the revised PCI that compensates for $K_{\delta}$, Figure $28 \mathrm{~b}$ shows the minimum PCI occuring at $K_{\delta}=0.16$, which is reasonably close to the value actually operated at. It is apparent from this brief analysis why encountering the jerk command dynamics compelled the pilot to utilize kinesthetic feedback - the cost was otherwise prohibitively high.

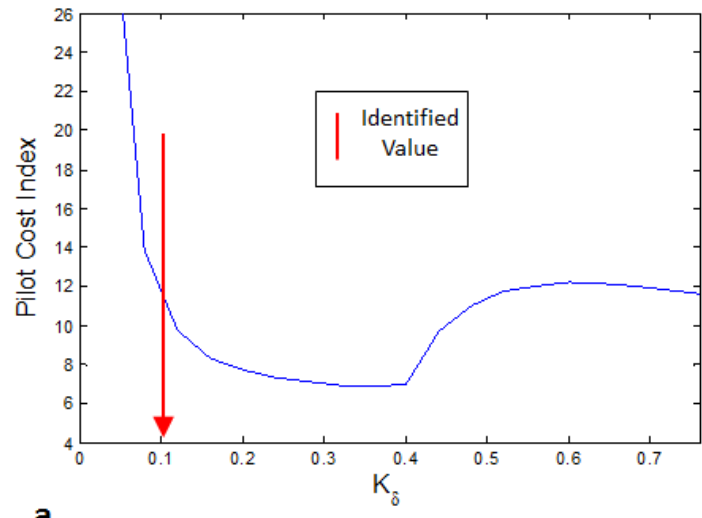

a.

Figure 28. Variation of Pilot Cost Index with proprioceptive feedback gain $\mathbf{K}_{\delta}$ (jerk command dynamics, Amplitude Clipping): a) No $K_{\delta}$ compensation (original PCI); b) With $K_{\delta}$ compensation (revised PCI).

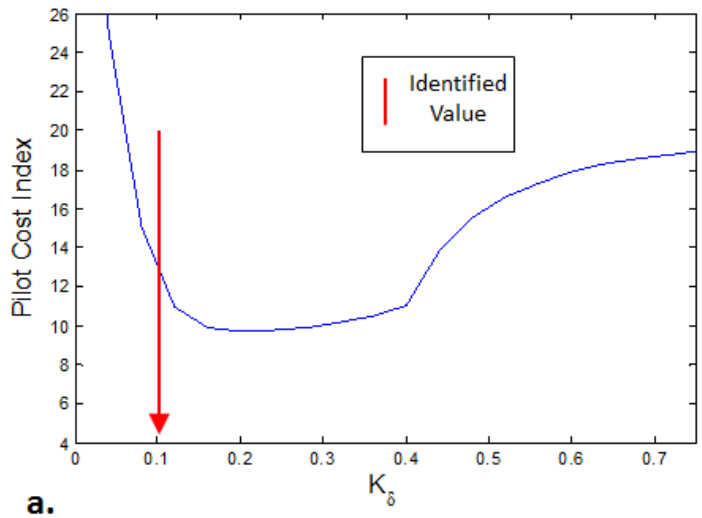

a. 
Reference to PCI will henceforth be regarding Eqn. 4, which accounts for $K_{\delta}$. Figure 29 shows the variation of PCI with the four modeled pilot parameters for the run examined in Figure 28 (jerk dynamics, amplitude clipping). The lower bound for $K_{e}$ (Figure 29a) is governed by the minimum variation in error the pilot can visually perceive. While the parameters selected by the pilot did not precisely correspond with the PCI minima of each parameter, the differences in PCI between selected and optimal were not very great. Thus it appears that the same PCI definition might be applied across very different vehicle dynamics for the same pilot. The question as to whether the other pilots shared a similar PCI costing will be explored next.
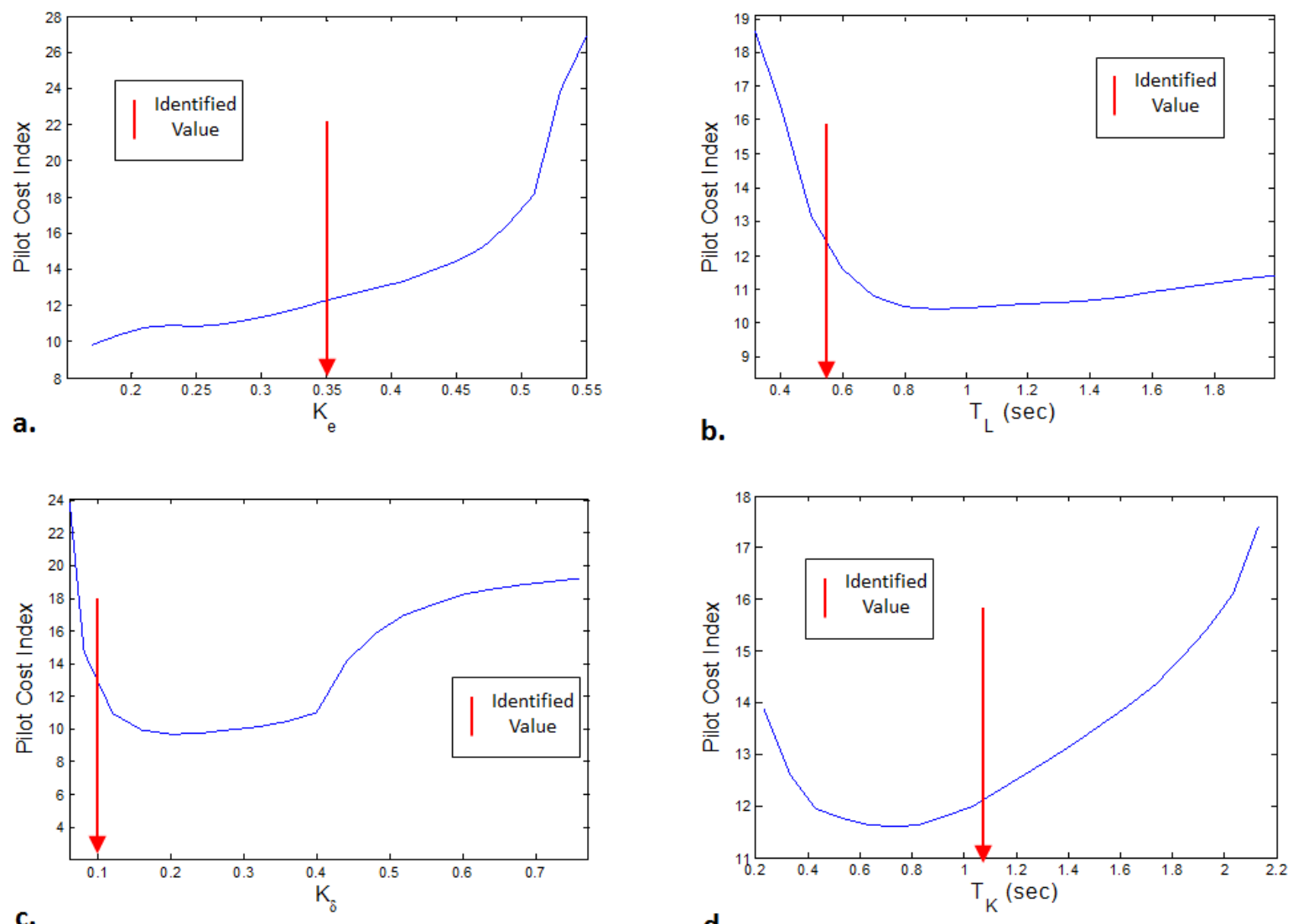

c.

Figure 29. Variation of Pilot Cost Index with pilot parameters (jerk command dynamics, Amplitude Clipping): a) $K_{e}$; b) $T_{L}$; c) $K_{\delta}$; d) $T_{K}$. 
Figure 30 examines PCI variation with pilot parameters for the PWM style (acceleration command run). Due to pulsing, some of the parameters produce a jagged PCI response when they are varied (i.e., $K_{e}, T_{K}$ ). In general the settings chosen by the pilot correspond to the 'knee' of the PCI curves where the change in PCI compared to the change in parameter has appreciably decreased (or before it appreciably increases).
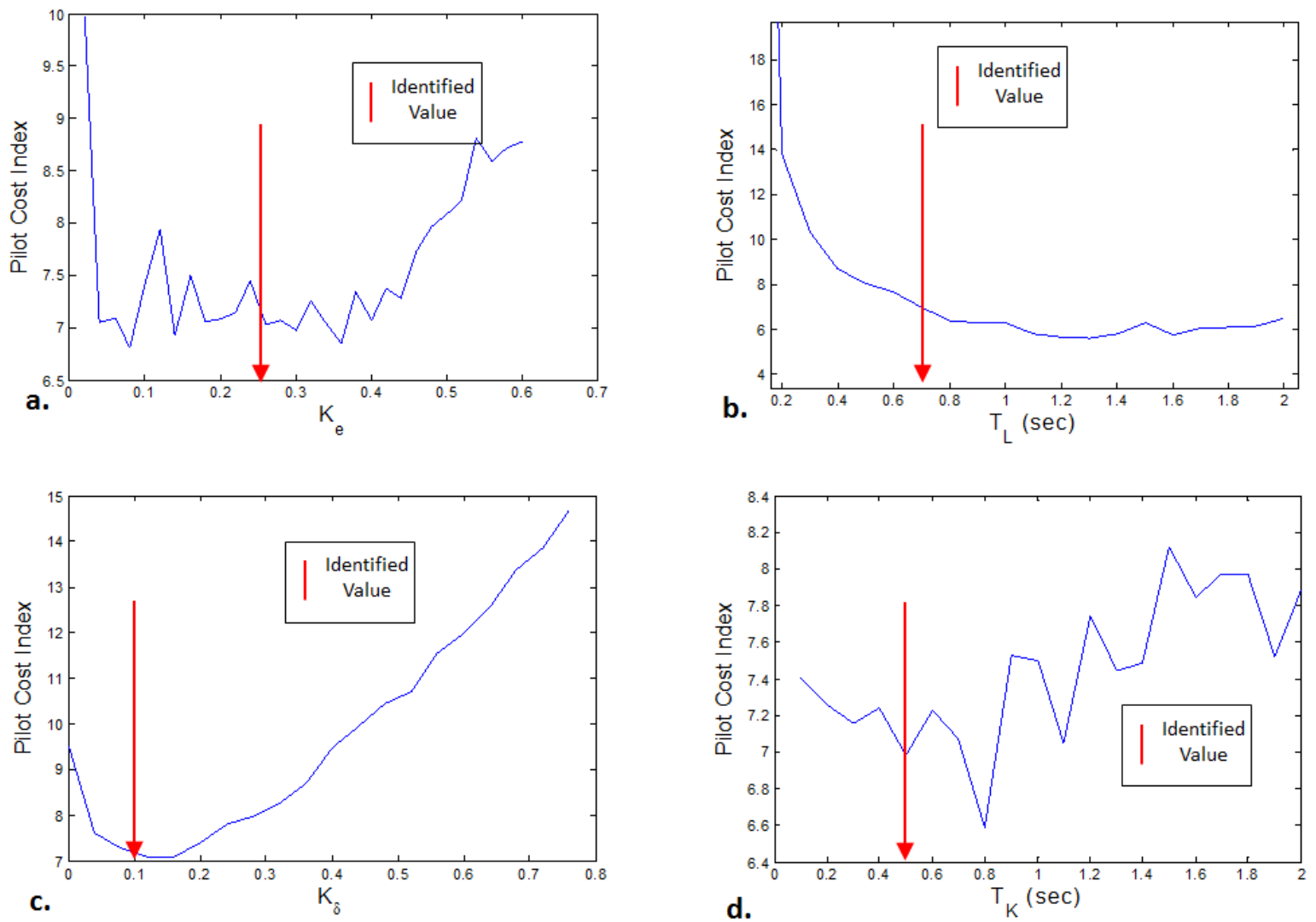

Figure 30. Variation of Pilot Cost Index with pilot parameters (acceleration command dynamics, Pulse Width Modulation): a) $K_{e}$; b) $T_{L}$; c) $K_{\delta}$; d) $T_{K}$. 
Figure 31 examines PCI variation with pilot parameters for the PWAM style (acceleration command run). Again, due to pulsing, some of the parameters produce a jagged PCI response when they are varied. Parameters selected by the pilot are proximal to the PCI minima.

Summarizing the observed PCI behavior, PCI was most strongly affected by $K_{\delta}$ in all of the four runs (small changes in $K_{\delta}$ brought about large changes in PCI). Significantly, the pilots' $K_{\delta}$ selections coincided closesly with the PCI minima for $K_{\delta}$. The lead time constant $T_{L}$ had the second largest impact of the four parameters. The selected values for $T_{L}$ were consistently less (but not appreciably) than the PCI minima locations, indicating that adding a term containing $T_{L}$ to the PCI definition in Eqn. 4 may be appropriate. The vehicle dynamics have a profound effect on PCI, such that the high operational penalties associated with jerk command drastically narrowed the range of feasible parameters.
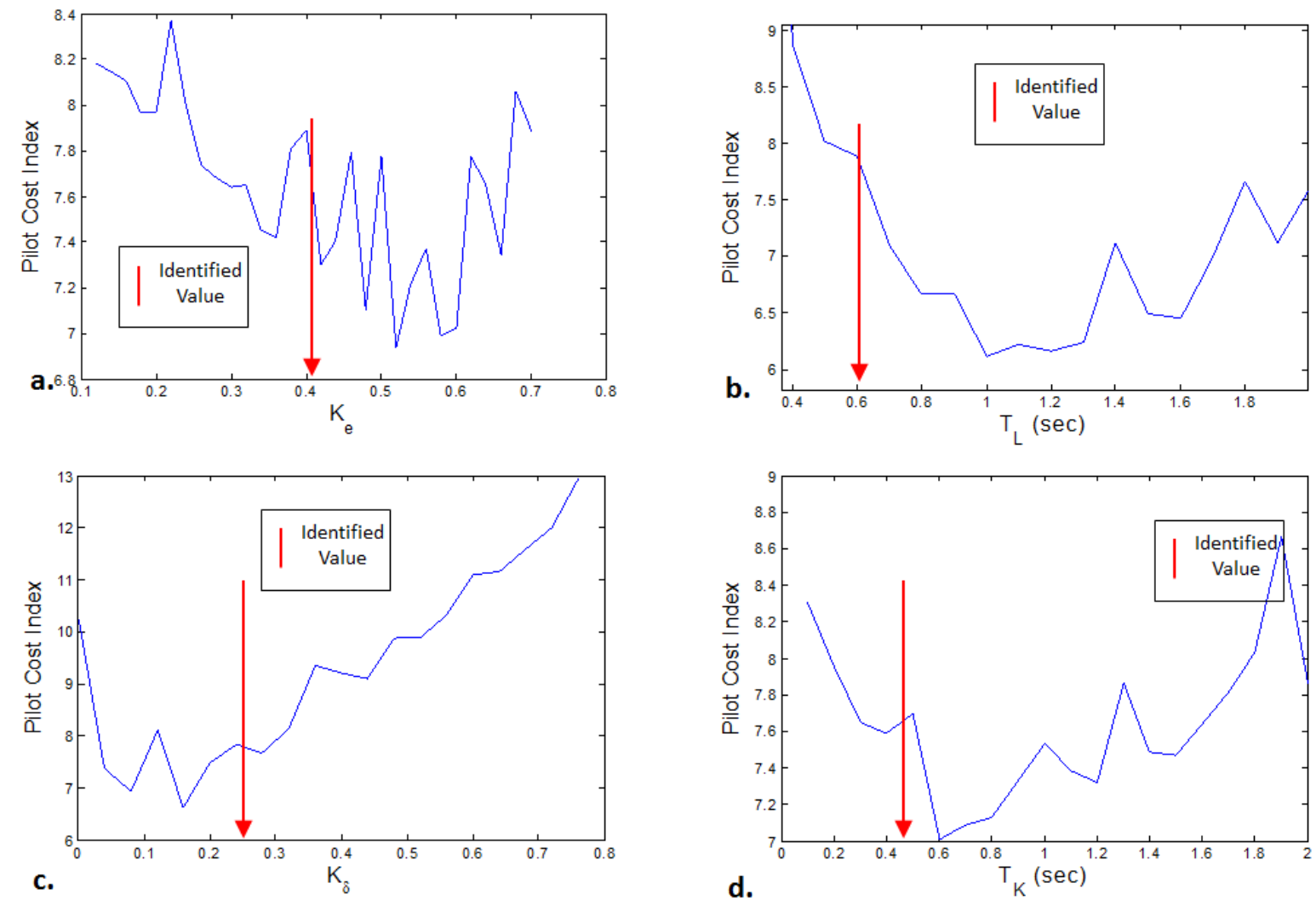

Figure 31. Variation of Pilot Cost Index with pilot parameters (acceleration command dynamics, Pulse Width Amplitude Modulation): a) $K_{e}$; b) $T_{L}$; c) $K_{\delta}$; d) $T_{K}$. 


\section{Global optimality}

Thus far, parameters have been varied about the 'trim' point (the parameter values identified in a pilot for a given run). Would successive iteration on parameter values which were chosen at their local minima tend toward a global minimum? Figure 32 investigates this by perturbing the trim point and comparing the resulting minimum PCI with the trim point's PCI. Figure 32a shows the nominal PCI response to $K_{\delta}$, with the minimum PCI of 6.2 occurring at $K_{\delta}=0$. In Figure $32 \mathrm{~b}$ the nominal $K_{e}$ is decremented by 0.05 , which yields an optimal $K_{\delta}=0.06$ (larger than the optimal $K_{\delta}$ using the nominal parameters) - but the minimum PCI associated with this is 6.6. Because of its larger PCI value, the off-nominal optimal $K_{\delta}$ would be rejected as a global minimum in the course of iteration. In Figure $32 \mathrm{c} K_{e}$ is incremented by 0.16 , producing an even larger PCI (7.3) associated with the optimal $K_{\delta}$. Finally in Figure $32 \mathrm{~d}$ the nominal $T_{L}$ is decremented by 0.5 , resulting in a very large optimal $K_{\delta}(0.2)$, but the associated PCI is 8.7 . The question of global optimality needs to be examined further, but this preliminary analysis appears encouraging.
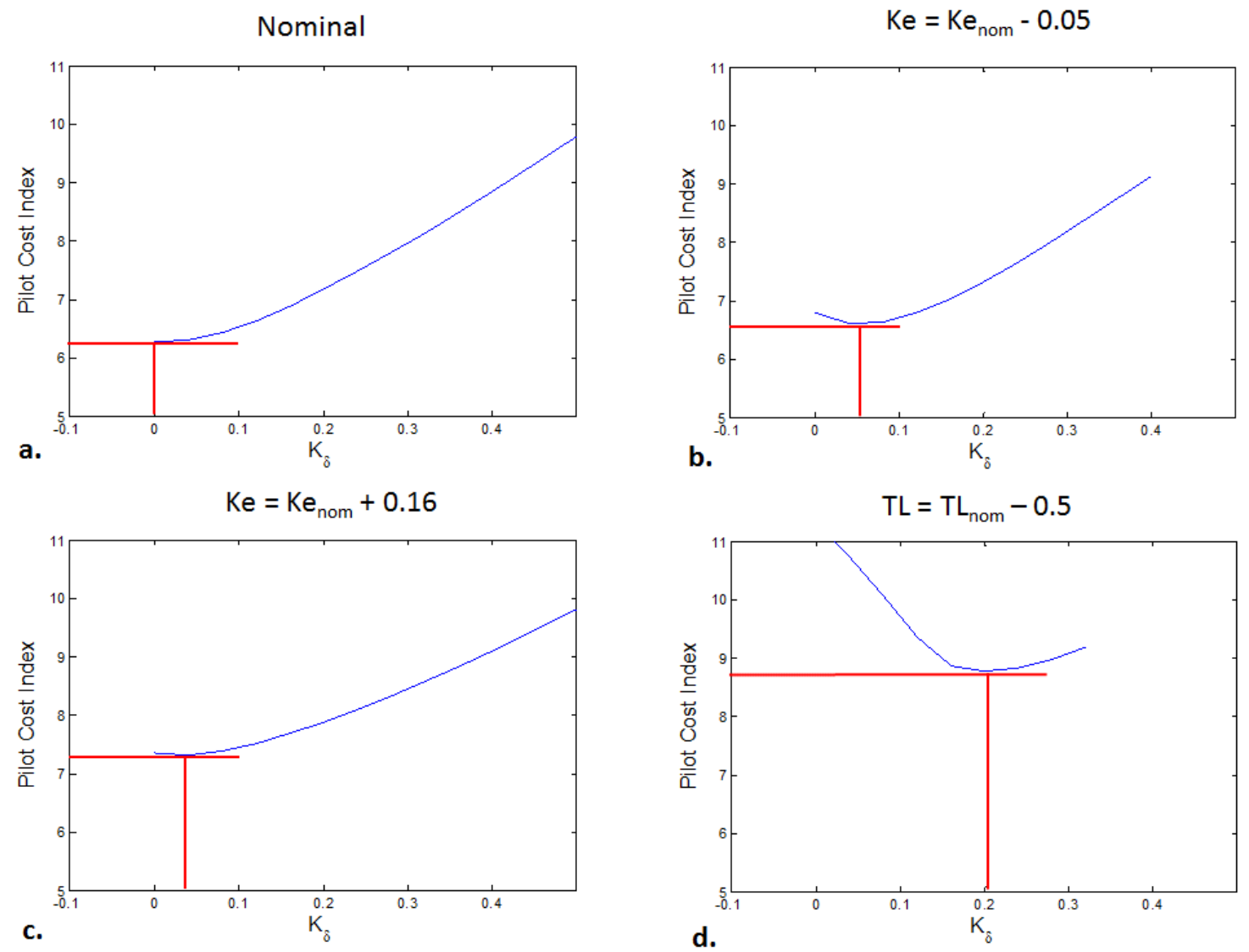

Figure 32. Variation of Pilot Cost Index with $K_{\delta}$ using off-nominal parameters (acceleration command dynamics, Amplitude Clipping): a) Nominal case; b) $K_{\mathrm{e}}$ decremented; c) $K_{\mathrm{e}}$ incremented; d) $T_{L}$ decremented. 
Figure 33 graphically presents the parameter optimization scheme that has been used in the discussions above. On the right, PCI is the summation of parameter costing (i.e. from $K_{\delta}$ and potentially $T_{L}$ ) with the product of $B_{\text {est }}$ and RMS error. For a given set of parameters, each parameter is individually varied to identify it local minimum, the result of which gives rise to a new optimal parameter of the set (if its PCI is smaller than the PCI of previous parameter value). This process occurs in the Parameter Optimization element. Also feeding into the Pilot Model is pitlot technique, task, and environment (the list is not exhaustive).

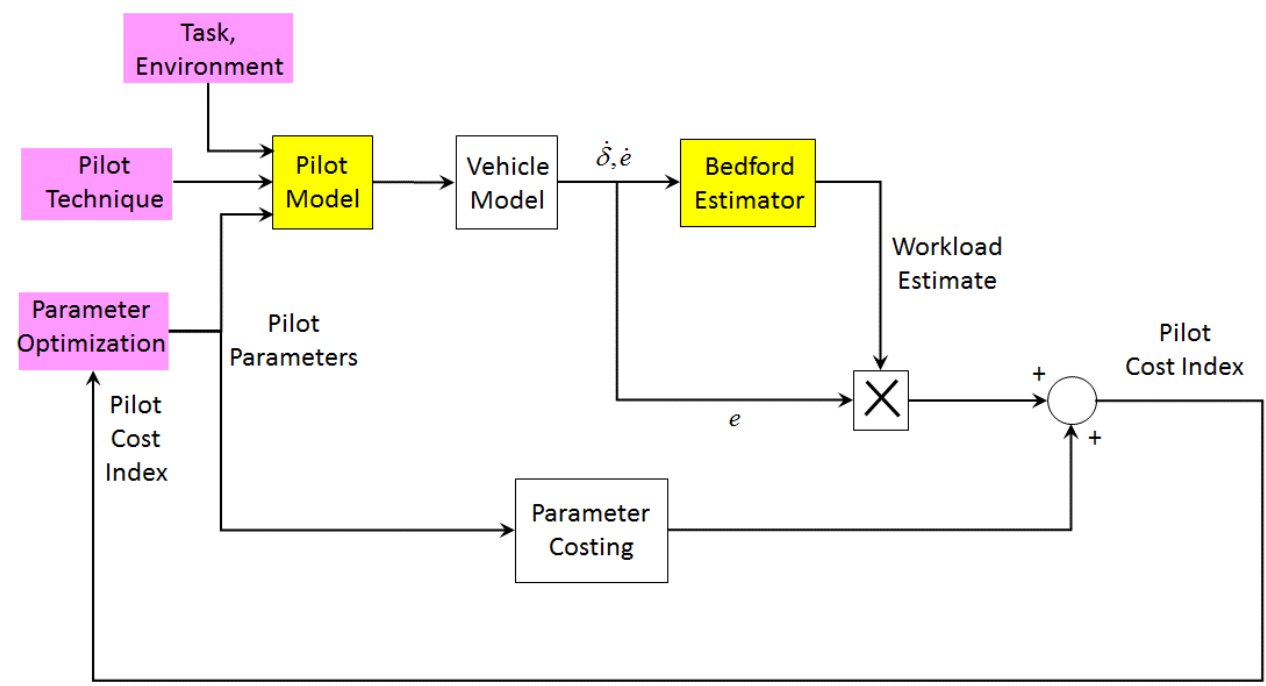

Figure 33. Procedure for computing pilot operating parameters.

\section{Conclusions}

Two models representing two types of observed pilot pulsive behavior - pulse width modulation (PWM), and pulse width amplitude modulation (PWAM) - were developed. These two pulsive models and a third model (amplitude-clipped continuous control) were analyzed using pilot data to establish if there is a systematic relationship between pilot workload and elements of Hess' pilot Structural Model. Preliminary results suggest:

1) The pulsive models used in conjunction with the pilot Structural Model closely reproduced the pilot data both in the frequency and time domains during closed-loop simulation. This suggests that for the range of tasks and control styles encountered, the models captured the fundamental mechanisms governing pulsive and control processes.

2) Pulsing can produce artificats such as low frequency droop that may appear as characteristics internal to the pilot when they are the result of control technique. Accurate modeling can identify such artifacts.

3) The pulse models developed give important insight for the amount of remnant (stick output uncorrelated with the forcing function) that arises from nonlinear pilot technique, and thus for the remaining remnant arising from different sources unrelated to tracking control (i.e. neuromuscular tremor, etc.).

4) In addition to emulating observed pilot behavior, the pilot Structural Model provides a method of elegance and economy for modeling higher frequency response. By assuming a relatively invariant pilot time delay and neuromuscular damping, and using the stick power spectrum to estimate the neuromuscular natural frequency, kinesthetic feedback produces systematic high frequency phase loss that is only coarsely accounted for by the single-loop Crossover Model with an effective time delay, attributed to increased level of difficulty.

5) During pulsive control of $\mathrm{K} / \mathrm{s}^{2}$ vehicle dynamics, it appears that skilled pilots: 1) produce a continuous, internally-generated stick signal that they integrated in time; 2) integrate the actual stick position; and 3) compare the two integrations to either issue or cease a pulse command. Since the two pulsive techniques (PWM and PWAM) required pure kinesthetic integration of the stick, it seems reasonable to assume that this same information could be leveraged by the first-order lag in the kinesthetic feedback loop. Both pulsive techniques were observed to employ this loop.

6) The pilot employing PWM rapidly deflected the gamepad's joystick to the stops (i.e. maximum deflection), producing sharply-edged and cornered pulses. The PWAM technique produced a train of spikes, where the 
stick impulsively rose to some peak amplitude and rapidly return to zero when released. The best matching between the modeled and observed behavior (PWM and PWAM) was obtained by setting the neuromuscular pilot element $Y_{N M}$ to unity, implying that those techniques were largely unaffected by the neuromuscular constraints typically associated with manual tracking. Thus pulsing can present a method for ameliorating unfavorable stick characteristics.

7) A Pilot Cost Index (PCI) has been developed which is comprised of workload, performance, and the degree to which the pilot employs kinesthetic feedback. Using this metric, operating ranges for the four pilot parameters were estimated and corresponded well with actual (measured) parameter values they used.

8) Workload rating reflects the effort corresponding with the parameter set employed. The PCI metric is composed of three observable measures - control rate and error rate (comprising the Bedford estimate), and error (performance) - and an inferred measure of pilot aversion to employ kinesthetic feedback (i.e. keep the feedback gain $K_{\delta}$ low). The same pilot who only used the error channel while controlling the $\mathrm{K} / \mathrm{s}^{2}$ plant was observed to employ kinesthetic feedback when encountering the substantially more difficult $\mathrm{K} / \mathrm{s}^{2}(\mathrm{~s}+\lambda)$ plant. The same definition of PCI was applied to all three pilots and yielded favorable results, preliminarily implying that skilled pilots possess similar internal 'costing' that determines parameter settings.

9) Control style (i.e., PWM, PWAM, continuous, etc.) may drive PCI and thus parameter selection, suggested by the observation that PWM and PWAM always employed proprioceptive feedback. Initial results indicate that parameter settings used by a pilot may correspond to a global PCI minimum rather than just a local minimum that was observed in the vicinity of an operating point. Thus it may be possible to predict likely parameter ranges for a given style, display sensitivity, and plant dynamics.

10) Past attempts at assigning a rating or a rating increment to individual parameter values or a ratio of parameters have met with limited success. An operator will probably never vary just one parameter and while the others go unchanged - if one varies the others will likely shift as well. For a given operational configuration (technique, skill level, display, vehicle, environment, etc.), the pilot should tend toward a set of internal settings that minimizes some cost index. This implies that a piecemeal approach is unsuitable - if the vehicle dynamics are altered, (or the display or control gains), the pilot adopts a different set of parameter values, and they shift in concert. The preliminary results suggest that pilots select their operating point based on minimizing the PCI, which indicates promise for evaluating and predicting pilot parameter selection for any existing or proposed system that requires manual control of the system's states. Additionally, this approach may be a means for investigating the viability of new control techniques and/or inceptor technologies.

The cases explored in this study are too few to offer statistically significant results, rather they are intended to provide insight and guidance for future research.

\section{Acknowledgments}

This work was supported by cooperative agreement NNX16AJ91A between the U.S. Army Aviation Development Directorate and San Jose State University. This paper has been approved for public release: unlimited distribution.

\section{References}

1 Hess, R. A., "Unified Theory for Aircraft Handling Qualities and Adverse Aircraft-Pilot Coupling", Journal of Guidance, Control, and Dynamics, Vol. 20, No. 6, 1997.

2 Tustin, A., "The nature of the human operators response in manual control and its implication for controller design," J. Instn. Elect. Engrs, 94, 190, 1947.

3 McRuer, D. T. and Krendel, E. S., "Mathematical Models of Human Pilot Behavior," No. AGARDograph No. 188, November 1973.

4 Bachelder, E. N., and D. H. Klyde, "Wavelet-Based Analysis of Roll Ratchet Using a Flight Test Database," AIAA $2003-5692$ presented at AIAA Atmospheric Flight Mechanics Conference, Austin, TX, August 11-14, 2003.

5 Smith, R. H., "A Unified Theory for Pilot Opinion Rating, "Proceedings of the Twelfth Annual Conference on Manual Control, May 1976, pp. 542-558.

6 R. A. Hess, "A Dual-Loop Model of the Human Controller," J. Guidance Contr., vol. 1, pp. 254-260, July-Aug. 1978. 
7 Hess, R. A., "Pursuit Tracking and Higher Levels of Skill Development in the Human Pilot", IEEE Transactions on Systems, Man, and Cybernetics, Volume: 11, Issue: 4, April 1981.

8 Gaines, B., "Linear and Nonlinear Models of the Human Controller," International Journal of Man-Machine Studies (1969) 1, 333-360.

9 Krendel, E., McRuer, D., "A servomechanisms approach to skill development," J. Franklin Inst., vol. 269, pp. 24-42, Jan. 1960. 10 Edkins, C., "The prediction of pilot opinion ratings using optimal and suboptimal pilot models", Masters Thesis, Air Force Institute of Technology, 1994.

11 Hess, R., "Prediction of aircraft handling qualities using analytical models of the human pilot", NASA Technical Memorandum 84233, 1982.

12 Davidson, J., Schmidt, D., "Modified optimal control pilot model for computer aided design and analysis", NASA-TM-4384, 1992).

13 McRuer, M., A Neuromuscular Actuation System Model, IEEE TRANSACTIONS ON MAN-MACHINE SYSTEMS, VOL. MMS-9, NO. 3, SEPTEMBER 1968.

14 Hess, R. A., "A Rationale for Human Operator Pulsive Control Behavior," Journal of Guidance, Control, and Dynamics, vol. 2, pp. 221-227, May-June 1979.

15 Hess, R. A., "Unified Theory for Aircraft Handling Qualities and Adverse Aircraft-Pilot Coupling", Journal of Guidance, Control, and Dynamics, Vol. 20, No. 6, 1997.

16 Hart, S., Wickens, C., "Workload Assessment and Prediction," Springer Netherlands, 1990

17 Hopkin, V. D., "Human factors in air traffic control," London, Taylor \& Francis, 1995.

18 Cooper, G. E. and Robert P. Harper, J., "The Use of Pilot Rating in the Evaluation of Aircraft Handling Qualities," Tech. Rep. NASA TN D-5153, National Aeronautics and Space Administration, April 1969.

19 Roscoe, A. H., \& Ellis, G. A., "A subjective rating scale assessing pilot workload in flight. A decade of practical use. Royal Aerospace Establishment.” Technical Report 90019. Farnborough. UK: Royal Aerospace Establishment, 1990.

20 Reid, G., "The Subjective Workload Assessment Technique: A Scaling Procedure for Measuring Mental Workload," Advances in Psychology, Volume 52, Pages 185-218, 1988.

21 Hart, S., "Development of NASA-TLX (Task Load Index): Results of Empirical and Theoretical Research," Advances in Psychology, Volume 52, Pages 139-183, 1988.

22 Bachelder, E., Aponso, B.: Novel Estimation of Pilot Performance Characteristics, AIAA Atmospheric Flight Mechanics Conference, AIAA SciTech Forum, (AIAA 2017-1640)

23 Mitchell, D., Aponso, B.,"Reassessment and extensions of pilot ratings with new data", 17th Atmospheric Flight Mechanics, Portland,OR, 1990. 